\title{
Inhibition of ER stress-mediated apoptosis in macrophages by nuclear-cytoplasmic relocalization of eEF1A by the HIV-1 Nef protein
}

\author{
W Abbas ${ }^{1}$, KA Khan ${ }^{1}$, MK Tripathy ${ }^{1}$, I Dichamp ${ }^{1}$, M Keita ${ }^{1}$, O Rohr ${ }^{2}$ and G Herbein ${ }^{\star, 1}$
}

HIV-1 Nef protein has key roles at almost all stages of the viral life cycle. We assessed the role of the Nef/eEF1A (eukaryotic translation elongation factor 1-alpha) complex in nucleocytoplasmic shuttling in primary human macrophages. Nuclear retention experiments and inhibition of the exportin-t (Exp-t) pathway suggested that cytoplasmic relocalization of eEF1A, mediated by Exp-t, occurs in Nef-treated monocyte-derived macrophages (MDMs). We observed the presence of tRNA in the Nef/eEF1A complexes. Nucleocytoplasmic relocalization of the Nef/eEF1A complexes prevented stress-induced apoptosis of MDMs treated with brefeldin-A. Blockade of stress-induced apoptosis of MDMs treated with HIV-1 Nef resulted from enhanced nucleocytoplasmic transport of eEF1A with decreased release of mitochondrial cytochrome $c$, and from increased tRNA binding to cytochrome $c$, ultimately leading to an inhibition of caspase activation. Our results indicate that HIV-1 Nef, through the nucleocytoplasmic relocalization of eEF1A and tRNAs, enhances resistance to stress-induced apoptosis in primary human macrophages.

Cell Death and Disease (2012) 3, e292; doi:10.1038/cddis.2012.32; published online 5 April 2012

Subject Category: Immunity

The endoplasmic reticulum (ER) is a well-orchestrated protein-folding machine composed of protein chaperones, proteins that catalyze protein folding, and sensors that detect the presence of misfolded or unfolded proteins. The unfolded protein response (UPR) is an intracellular signaling pathway that coordinates ER protein-folding demand with proteinfolding capacity and is essential to adapt to homeostatic alterations that cause protein misfolding. ${ }^{1}$ Accumulating evidence suggests that protein folding and generation of reactive oxygen species (ROS) as a byproduct of protein oxidation in the ER are closely linked events. It has also become apparent that activation of the UPR on exposure to oxidative stress is an adaptive mechanism to preserve cell function and survival. ${ }^{1}$ Persistent oxidative stress and protein misfolding initiate apoptotic cascades and are now known to have predominant roles in the pathogenesis of multiple human diseases, including diabetes, atherosclerosis and neurodegenerative diseases. $^{2}$

Excessive ROS production not appropriately compensated by antioxidant molecules can lead to oxidative stress, which may have an important role in pathogenesis of HIV infection through various mechanisms. ${ }^{3-5}$ In monocytes from asymptomatic patients, the levels of $\mathrm{Bcl}-2$ and thioredoxin (TRX) decrease, ${ }^{4}$ which is associated with enhanced hydrogen peroxide production, whereas in cells from AIDS patients the levels returned to normal. ${ }^{6}$ Thus, the redox balance affected by the intracellular TRX system seems to be important for cell survival and low viral production, probably leading to chronic persistent infection of HIV. HIV-1 Tat has been reported to induce UPR, ${ }^{7}$ indicating that ER stress response could be a critical parameter to control during HIV infection.

The translation elongation factor EF1A has been described as a cytoplasmic component of the nuclear aminoacylationdependent tRNA export pathway. ${ }^{8}$ Eukaryotic translation elongation factor 1-alpha (eEF1A) interacts directly with aminoacylated tRNAs. Exportin-t (Exp-t) has been reported to have a major role in eEF1A-mediated export, although Exp-5, which preferentially exports pre-miRNAs, has been reported to be involved in the nuclear-cytoplasmic transport of tRNAs in some cases. ${ }^{9}$ In addition to the conventional role of eEF1A during protein synthesis, namely GTP-dependent binding and transport of aminoacyl-tRNA to the A site of the ribosome, eEF1A has a role in the cytoplasm. One of the proposed unconventional functions of eEF1A is a role in the regulation of cytoskeletal dynamics. ${ }^{10}$ In addition to binding GDP/GTP, aminoacyl-tRNA, EF1 $\beta$ and the ribosome, eEF1A binds and bundles actin and binds to microtubules. Because eEF1A is an abundant protein in most eukaryotic cells and binds to actin filaments with relatively high affinity, it could be a potent regulator of the cytoskeleton. ${ }^{11}$ Previous in vitro

\footnotetext{
${ }^{1}$ Department of Virology, University of Franche-Comte, EA 4266, INSERM IFR 133, CHU Besancon, Besançon F-25030, France and ${ }^{2}$ Institut de Parasitologie et Pathologie Tropicale, EA 4438, Strasbourg University, 3 rue Koeberlé, Strasbourg 67000, France

*Corresponding author: G Herbein, UPRES EA4266 'Pathogens \& Inflammation', Department of Virology, University of Franche-Comte, CHU Besancon, 2 place SaintJacques, Besançon F-25030, France. Tel: + 333812188 77; Fax: + 333816656 95; E-mail: georges.herbein @ univ-fcomte.fr Keywords: Nef; eEF1A; tRNA; cytochrome $c$; apoptosis; macrophage

Abbreviations: ASK-1, apoptosis signal-regulating-kinase 1; BFA, brefeldin-A; eEF1A, eukaryotic translation elongation factor 1-alpha; ER, endoplasmic reticulum; Exp-t, exportin-t; MDMs, monocyte-derived macrophages; PBLs, peripheral blood lymphocytes; PBMCs, peripheral blood mononuclear cells; ROS, reactive oxygen species; TG, thapsigargin; TM, tunicamycin; TRX, thioredoxin; Try or Tryp, tryptophan; UPR, unfolded protein response

Received 15.11.11; revised 27.2.12; accepted 29.2.12; Edited by A Stephanou
} 
studies demonstrated that eEF1A inhibits the rate of actin polymerization and stabilizes actin filaments. ${ }^{12}$

In addition, eEF1A has been reported to have a role in apoptosis or programmed cell death. Early experiments showed that the level of eEF1A expression in cultured mouse fibroblasts correlates with the rate of apoptosis on serum withdrawal, with higher levels of eEF1A expression associated with a faster rate of cell death. ${ }^{13}$ Other studies have indicated that eEF1A prevents cell death, and numerous studies have shown that eEF1A expression increases in tumor cells and tumor tissues parallel with decreased caspase-3 activation. ${ }^{14,15}$

Nef a $27-k D a$ HIV-1 protein is translated from multiply spliced viral mRNAs early during infection. ${ }^{16}$ Endogenous Nef may have evolved a number of different, independent functional activities to enhance the replication and survival of the virus within infected cells and to facilitate its spread in vivo. ${ }^{17}$ Nef enhances virion infectivity and increases viral replication in primary lymphocytes and macrophages. ${ }^{18}$ The protein can also mediate downregulation of CD4 cell surface expression, a phenomenon important for the release of HIV-1 from the cell. ${ }^{17}$ In addition, Nef can downregulate the cell surface expression of major histocompatibility complex class I molecules, an effect found to protect infected cells from cytotoxic T cells. ${ }^{19}$ The Nef protein prevents apoptosis of HIV1 -infected $T$ cells through either interference with Fas/TNF $\alpha$ receptor death signaling pathways by inhibiting apoptosis signal-regulating-kinase 1 (ASK-1) ${ }^{20}$ or the formation of a complex with both p21-activated kinase and phosphatidylinositol-3-kinase, which increases phosphorylation and inactivation of the proapoptotic Bad protein. ${ }^{21}$ HIV-1 Nef protects human monocyte-derived macrophages (MDMs) from HIV-1induced apoptosis, and this protection correlates with the hyperphosphorylation and consequent inactivation of Bad. ${ }^{22}$ Nef expression within macrophages has been reported to favor the recruitment of resting $T$ cells via the secretion of $C-C$ chemokines and to subsequently favor their activation, suggesting a role for Nef in lymphocyte recruitment and activation at sites of viral replication. ${ }^{23}$

Our results indicate that HIV-1 Nef associates with eEF1A and that Exp-t contributes to the nuclear-cytoplasmic transport of Nef/eEF1A/tRNA complexes in macrophages. Finally, we observed that cytoplasmic relocalization of the Nef/eEF1A/tRNA complexes prevents stress-induced apoptosis in macrophages via increased cytoplasmic eEF1A expression, decreased release of mitochondrial cytochrome $c$ and plugging of released cytochrome $c$ by cytoplasmic tRNAs, ultimately resulting in decreased caspase activation.

\section{Results}

Identification of the interaction between HIV-1 Nef and eEF1A. In order to identify potential HIV-1 Nef protein/ protein interactions, we screened high-density protein expression filter membranes containing 55296 clones from a human fetal brain library using Far-western analysis with recombinant HIV-1 Nef as bait. ${ }^{24}$ We identified eEF1A as a potential binding candidate (Figure 1a). To test this interaction, we expressed a Nef-GST (glutathione S-transferase) fusion protein in $E$. coli and tested its ability to interact with eEF1A from U937 cell lysates. The Nef protein bound to eEF1A (Figure 1b). Endogenous eEF1A protein present in the lysates of Vero cells, MRC5 cells, promonocytic U937 cells, primary peripheral blood lymphocytes (PBLs) and primary MDMs co-immunoprecipitated with recombinant Nef (rNef) added to the culture, whereas the isotype control showed no associated eEF1A protein on immunoprecipitation (Figure 1c). Although the interaction between eEF1A and $\mathrm{rNef}$ was detected in both nuclear and cytoplasmic lysates prepared from the cell lines (Vero cells, MRC5 cells, U937 cells), we measured more eEF1A/rNef complexes in the nucleus than in the cytoplasm of primary MDMs and PBLs early post-treatment (30 min; Figure 1c).

The interaction between eEF1A and endogenous Nef was also demonstrated by transient transfection of U937 cells with a nef-expressing plasmid (Figure 1d), and in peripheral blood mononuclear cells (PBMCs) infected in vitro with HIV-189.6 (Figure 1e). Thus, eEF1A interacts with the Nef protein, not only in vitro within several cell types treated with $\mathrm{rNef}$, but also with the endogenous Nef protein produced within HIV-1infected primary PBMCs. Lysates from MDMs treated with

\footnotetext{
Figure 1 eEF1A interacts with HIV-1 Nef protein in vitro and in vivo. (a) Image analysis of a high-density protein filter array screened by nonradioactive Far-western blotting using HIV- $1_{89.6}$ Nef as a probe. A magnified section of the blot is shown, indicating the interacting double-spotted eEF1A clones (black). Guide dots (white) on the highdensity filter membrane facilitate identification of the $x y$-coordinates of positive clones. (b) Binding of HIV-1 Nef to eEF1A was measured in GST pull-down assays using U937 cells as a source of lysates. Input corresponds to $10 \%$ of the material used for pull-down. $\beta$-Actin detection represents input loading controls of the lystates that were used in binding reactions. Results are representative of three independent experiments. The right panel represents Commassie staining of expressed proteins that were used in the binding reaction of GST pull-down. (c) Cytoplasmic and nuclear extracts from several cell lines (Vero, MRC5, U937 cells), PBLs, and MDMs treated with rNef (100 ng/ml) for $0.5 \mathrm{~h}$ were immunoprecipitated with an anti-eEF1A antibody, or Nef $(100 \mathrm{ng} / \mathrm{ml})$-treated total cellular lysates were immunoprecipitated with an isotype control antibody. Immunoprecipitated material was analyzed by western blotting with an anti-Nef monoclonal antibody. Recombinant Nef-treated total cell lysates were used as a positive control. Results are representative of three independent experiments. (d) Cellular extracts of MDMs transfected for $48 \mathrm{~h}$ with a nef-expressing plasmid (pNef) were immunoprecipitated with an anti-eEF1A antibody, anti-Nef antibody, or isotype control antibody. Immunoprecipitated material was analyzed by western blotting with an antiNef monoclonal antibody. Results are representative of two independent experiments. (e) Cellular extracts of PBMCs infected in vitro with HIV-1 89.6 or mock infected were immunoprecipitated with an anti-eEF1A antibody or anti-Nef monoclonal antibody. Immunoprecipitated material was analyzed by western blotting with an anti-Nef monoclonal antibody or anti-eEF1A antibody. Results are representative of two independent experiments. (f and $\mathbf{g})$ eEF1A and HIV-1 Nef interact in vitro in a mammalian two-hybrid assay. (f) Schematic representation of expression constructs used in co-transfection experiments in the mammalian two-hybrid model. (g) Mammalian two-hybrid analysis with eEF1A fused to the VP16 activator domain and HIV-1 Nef fused to the GAL4 domain. Luciferase assays were conducted on total extracts from U937 cells transfected with the luciferase expression construct pG5-Luc, pBIND-Nef, pACT-eEF1A or control plasmids. Results represent the average of a triplicate experiment in which luciferase was normalized to protein expression. As a positive control, two plasmids, pACT-MyoD and pBIND-Id, were co-transfected, and co-transfection of empty vectors was used as a negative control. Results represent the mean of three independent experiments. ${ }^{* \star *} P<0.001$
} 
rNef in vitro were immunoprecipitated with antibodies directed against eukaryotic translation elongation factor-2 (eEF2), and western blot performed with an anti-Nef antibody. The Nef protein did interact with eEF1A, but not with eEF2 (data not shown), indicating that the Nef/eEF1A interaction was specific. We further investigated the interaction between eEF1A and Nef using a mammalian two-hybrid assay. Nef fused to the GAL4 DNA-binding domain (pBIND) was used as the bait vector, and eEF1A fused to the VP16-activation domain ( $p A C T)$ was used as the prey vector (Figure 1f). The constructs were transfected into mammalian cells along with the pG5Luc vector, which contains five GAL4 binding sites upstream of a minimal TATA box upstream of the luciferase gene. The interaction between the GAL4-Nef and VP16eEF1A fusion constructs resulted in an 18-fold increase of relative luciferase expression over the negative control (1847 versus 105 relative light units (rlu); Figure 1g). Expression of pGAL4-Nef alone or pVP16-eEF1A alone did not result in increased luciferase activity compared with negative controls (115 and 103 rlu, respectively). a

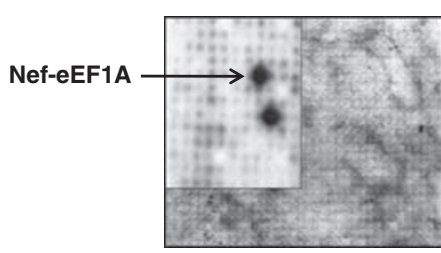

b

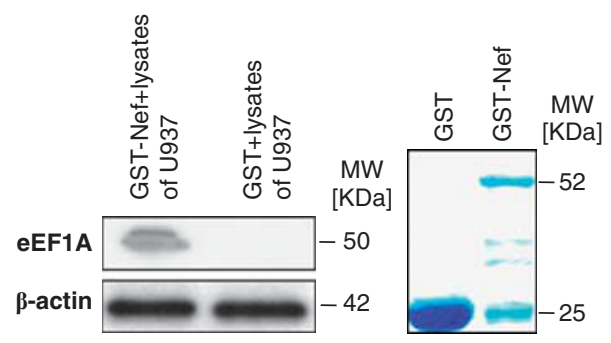

C

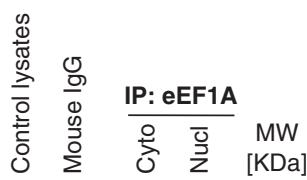

d

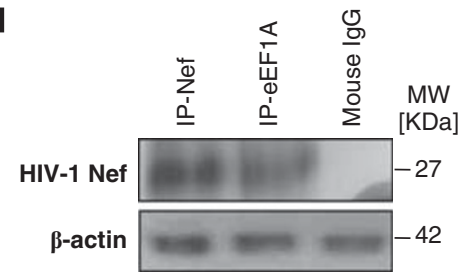

e

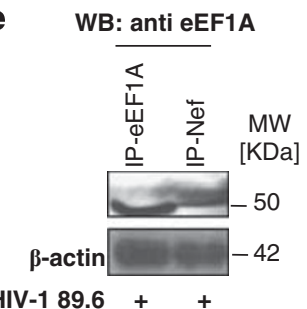

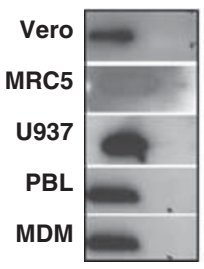

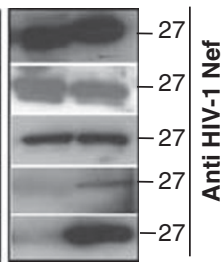

WB: anti Nef

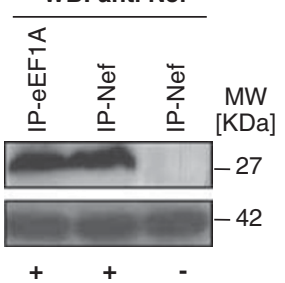

f
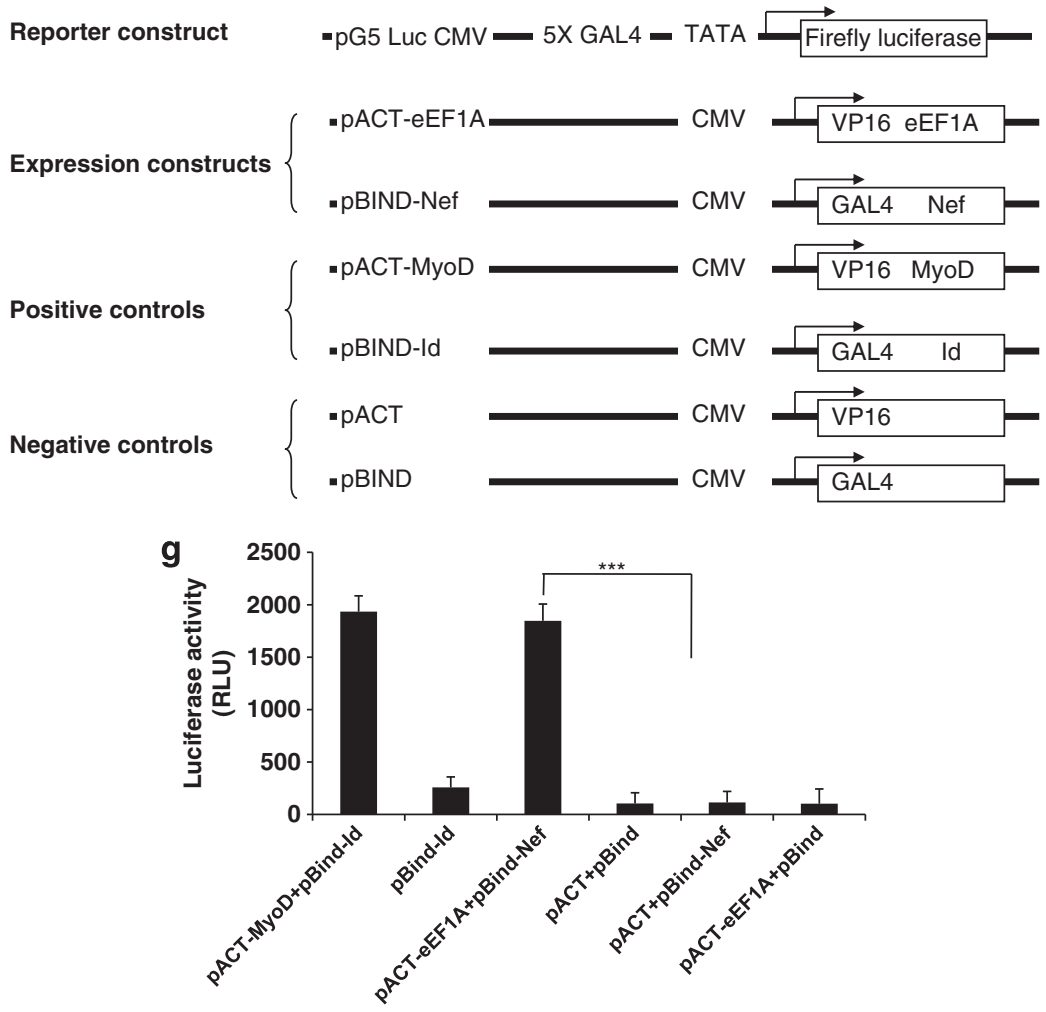
Determination of eEF1A and HIV-1 Nef regions involved in eEF1A-Nef interaction. To determine the region of eEF1A responsible for association with HIV-1 Nef, we used deletion mutants engineered within the cDNA encoding eEF1A. ${ }^{25}$ Lysates from bacteria expressing $\mathrm{N}$-terminal HAtagged eEF1A mutants were used in binding reactions with GST-HIV-1 Nef. The deletion mutants we analyzed are shown in Figure $2 a$. Three of the deletion mutants were not stably expressed (Figure 2a, bacterial expression). Of the mutants that were expressed, only the eEF1A 1-74 mutant was capable of binding Nef (Figure 2a). These results demonstrate that the eEF1A fragment encoding the $\mathrm{N}$-terminal 74 amino acids is sufficient for HIV-1 Nef binding.

To determine the region of HIV-1 Nef responsible for binding to eEF1A, two GST-Nef deletion mutants (C terminus, GST-Nef1-60; N terminus, GST-Nef55-206) were produced and assayed for their ability to associate with eEF1A in vitro (Figure 2b). The GST-Nef1-60 and GST-Nef55-206 mutants had intact $\mathrm{C}$-terminal and $\mathrm{N}$-terminal sequences (data not shown). Lysates from MDMs were used in binding reactions with GST-HIV-1 Nef constructs. Of the three GSTNef constructs, only GST-NefWT and GST-Nef55-206 were capable of binding eEF1A (Figure 2b). These results demonstrate that the $\mathrm{N}$ terminus of $\mathrm{Nef}$ is not required for eEF1A binding, and that the Nef fragment encoding amino acids 55 to 206 is sufficient.

Cytoplasmic relocalization of eEF1A by HIV-1 Nef is mediated by Exp-t. eEF1A protein is found in both the nucleus and cytoplasm of cells, which favors the nuclear export of mature tRNAs. In the absence of Nef, we observed much more eEF1A in the nucleus than the cytoplasm of primary MDMs (Figure 3a). To determine whether Nef, via its interaction with eEF1A, interferes with the nuclear-cytoplasmic distribution of eEF1A, we treated MDMs with rNef. ${ }^{26}$ Nuclear and cytoplasmic extracts were prepared from cells treated up to $12 \mathrm{~h}$ with $\mathrm{rNef}$, and the eEF $1 \mathrm{~A} / \mathrm{rNef}$ interaction was assessed in both cellular compartments. Recombinant Nef enhanced the nuclear-cytoplasmic transport of eEF1A up to $12 \mathrm{~h}$ post-treatment in MDMs (Figure 3a). Our results indicate that $r$ Nef could actively favor the nuclear export of eEF1A in MDMs.

We tested several exportins, Exp-t, Exp-1 and Exp-5, with regard to the nuclear-cytoplasmic transport of eEF1A in the presence of rNef. Using MDM lysates, we detected a direct interaction between GST-Nef and Exp-t using a pull-down assay (Figure 3b). No direct interaction between GST-Nef and Exp-1 was observed (data not shown). Using lysates from untreated MDMs, we detected an interaction between eEF1A and Exp-t by co-immunoprecipitation (Figure 3c). We did not detect any interaction between eEF1A and Exp-1 in untreated MDMs (data not shown).

Confirming an interaction between Exp-t and eEF1A or Nef, we observed a transient rNef/eEF1A/Exp-t complex in cytoplasmic extracts from MDMs treated with rNef $30 \mathrm{~min}$ post-treatment (Figure 3a). A sustained presence of rNef/ eEF1A complexes was detected in the cytoplasm of rNeftreated MDMs up to $12 \mathrm{~h}$ post-treatment (Figure 3a). To determine the role of Exp-t in the nuclear-cytoplasmic transport of $\mathrm{rNef} / \mathrm{eEF} 1 \mathrm{~A}$ complexes, MDMs were transfected with Exp-t siRNA $48 \mathrm{~h}$ prior to treatment with rNef. Knockdown of the Exp-t protein in MDMs was monitored by western blot (Figure 3d). Knockdown of Exp-t resulted in enhanced nuclear retention of eEF1A and $r$ Nef in MDM cultures treated with $r$ Nef (Figure $3 e$ ). We did not detect any nuclear retention of $r$ Nef or eEF1A following treatment of MDMs with the Exp-1 inhibitor leptomycin B or Exp-5 siRNA (data not shown). Our results indicate that, in rNef-treated MDMs, the nuclear-cytoplasmic transport of rNef/eEF1A complexes is preferentially mediated by Exp-t.

Nuclear-cytoplasmic relocalization of eEF1A by rNef blocks stress-induced apoptosis in macrophages. Brefeldin-A (BFA) disrupts the organization of the microtubule and actin cytoskeleton and exerts ER and Golgi stress, resulting in apoptosis (Supplementary Figure 1). Interestingly, eEF1A is an actin/microtubule binding protein and could interfere with BFA-induced apoptosis. Therefore, we examined whether nuclear-cytoplasmic transport of eEF1A by rNef inhibits BFAinduced apoptosis in MDMs, potentially due to cytoplasmic accumulation of eEF1A. We observed that pretreatment with rNef inhibits BFA-induced apoptosis in MDMs in a dosedependent manner (Figure 4a). In addition, pretreatment with rNef did not inhibit apoptosis induced by tunicamycin (TM) or thapsigargin (TG) that disturb protein glycosylation or $\mathrm{Ca}^{2+}$ signaling, respectively (Figure 4b). Of the three Nef proteins, only NefWT and Nef55-206 were capable of blocking BFAinduced apoptosis in MDMs (Figure 4c).

Because Exp-t is involved in nuclear-cytoplasmic transportation of the eEF1A/rNef complexes, we assessed apoptosis among MDMs treated with rNef prior to BFA treatment and transfected with siRNA Exp-t, siRNA Exp-1, siRNA Exp-5 or scramble control. We observed that transfection with siRNA Exp-t, but not siRNA Exp-1 or siRNA Exp-5, inhibited the rNefmediated blockade of apoptosis in MDMs treated with BFA (Figure 4d). Our results indicate that Exp-t-mediated nuclearcytoplasmic transport of the rNef/eEF1A complexes in MDMs is involved in the inhibition of apoptosis triggered by stress, such as BFA treatment.

As shown in Figure 5a, p35 procaspase- 3 is diminished and cleaved to $\mathrm{p} 20 / \mathrm{p} 17$ proteins in MDMs treated with BFA, confirming that BFA-induced apoptosis activates caspase3. 27,28 Under ER stress treatments, such as BFA, the magnitude of protection against apoptosis directly correlates with the level of cytoplasmic eEF1A expression ${ }^{29,30}$; therefore, we measured the levels of eEF1A in the cytosol of BFAstimulated MDMs treated with increasing concentrations of Nef. We observed that the levels of cytosolic eEF1A positively correlated with Nef treatment in a dose-dependent manner, as well as with the resistance to apoptosis in BFA-stimulated MDMs as measured by decreased caspase- 3 activation (Figure 5b).

Cytoplasmic extracts of BFA-stimulated MDMs treated with rNef, in the absence or presence of Exp-t siRNA, Exp-1 siRNA or Exp-5 siRNA, were analyzed for caspase-3 activation. Exp-t, Exp-1 and Exp-5 knockdown in MDMs was monitored by western blot (Figures 3d and 5c). Knockdown of Exp-t, but not Exp-1 or Exp-5, relieved the Nef-mediated blockade of apoptosis in BFA-stimulated MDMs (Figure 5d). Relief of the Nef-mediated blockade of apoptosis in BFA-stimulated MDMs 
a

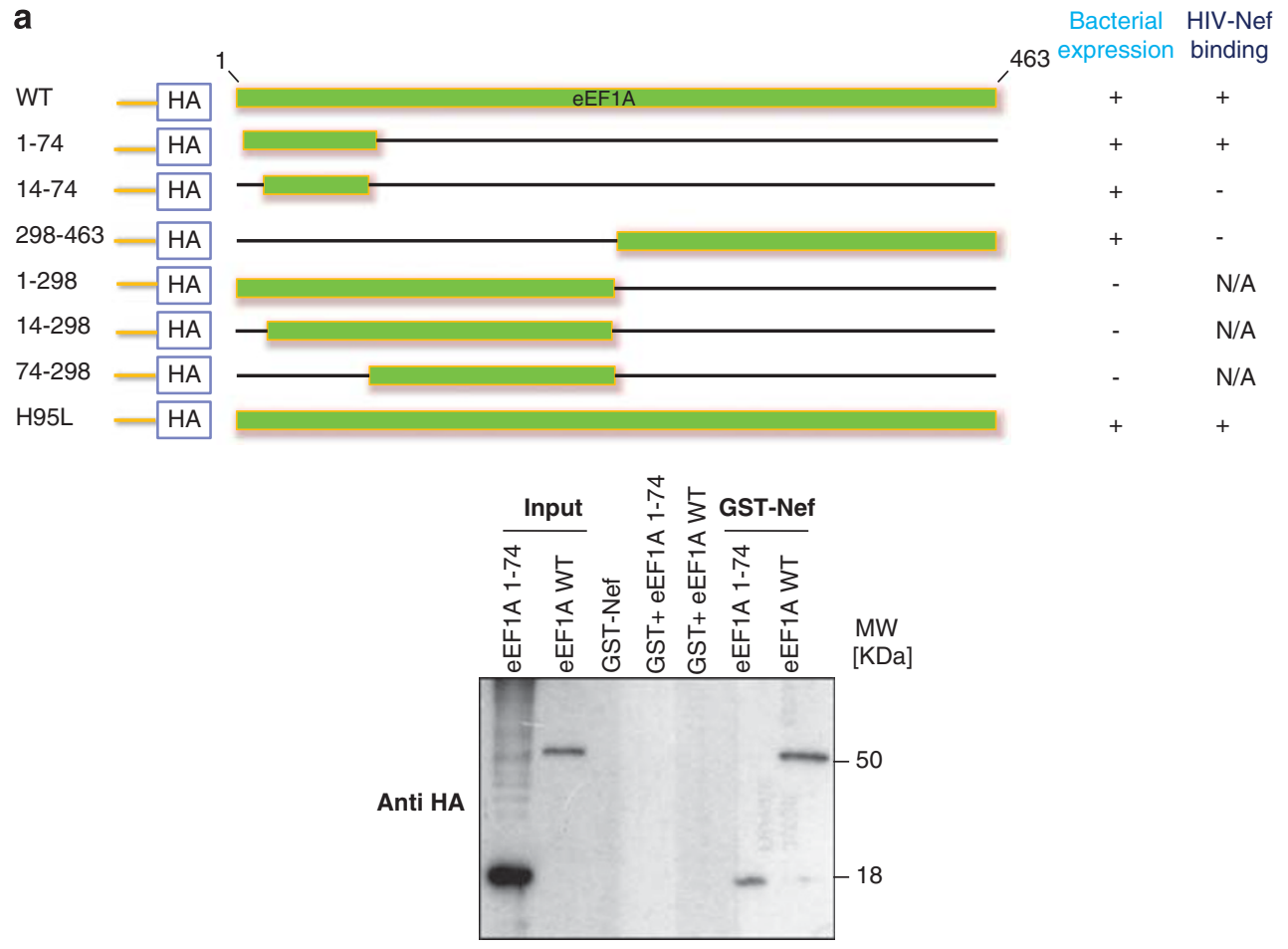

b

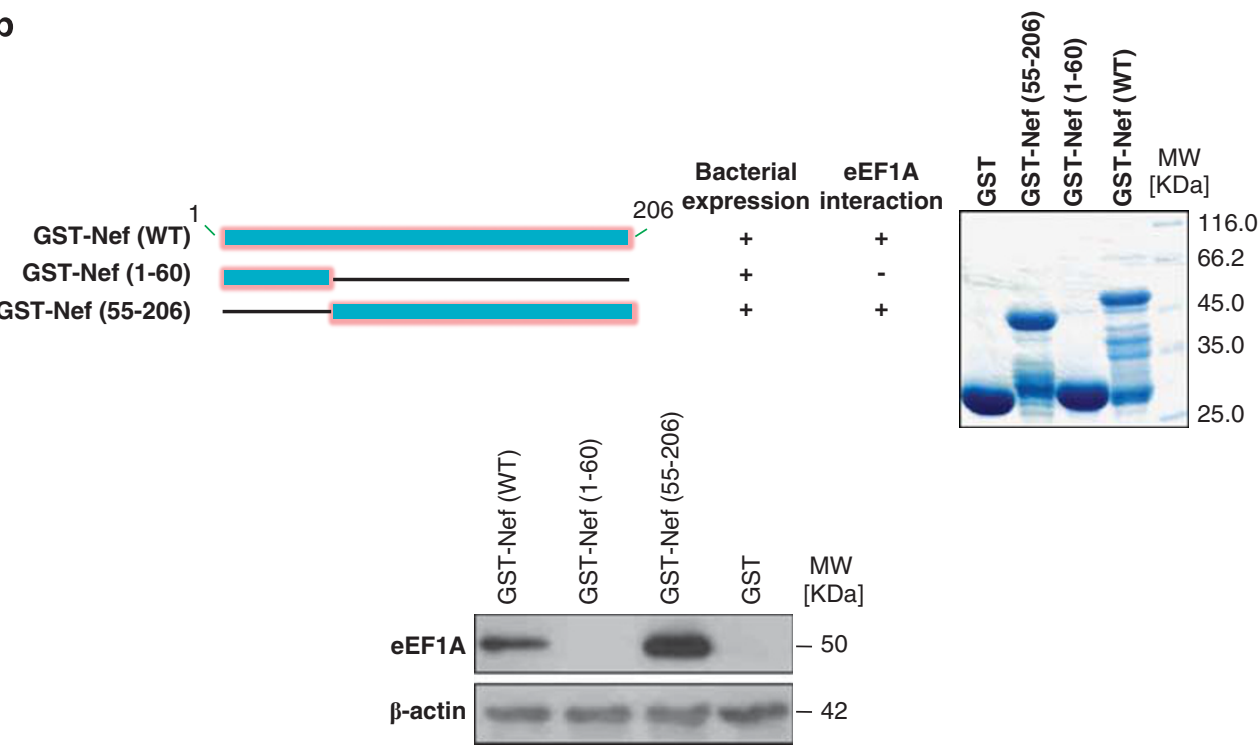

Figure 2 The N-terminal 74 amino acids of eEF1A binds to the C-terminal region (aa 55-206) of HIV-1 Nef. (a) The N-terminal region is sufficient for binding to HIV-1 Nef. Upper panel, Schematic diagram of eEF1A mutants expressed in bacteria as HA-tagged fusion proteins. The names of the mutants are shown to the left of the figure; numbers refer to the amino acid residues retained by the deletion mutants. The bacterial expression status and in vitro binding of these mutants are reported qualitatively as + or - to the right of the figure. NA, not applicable; WT, wild type. Lower panel, eEF1A interacts with HIV-1 Nef via its N terminus extremity (aa 1-74). Using wild-type GST-Nef constructs, binding of purified WT eEF1A and eEF1A 1-74 was measured in GST pull-down assays. Input corresponds to $10 \%$ of the material used for pull-down. Results are representative of two independent experiments. (b) The C-terminal region (aa 55-206) of HIV-1 Nef is sufficient for binding to eEF1A. Upper left panel, Schematic diagram of WT HIV-1 Nef and mutants expressed in bacteria as GST-tagged fusion proteins. The names of the mutants are shown to the left of the figure; numbers refer to the amino-acid residues retained by the deletion mutants. The bacterial expression status and in vitro binding of these mutants are reported qualitatively as + or - to the right of the figure. Upper right panel represents Commassie staining of expressed proteins that were used in the binding reaction of GST pull-down. Lower panel, HIV-1 Nef interacts with eEF1A via its C-terminal region (aa 55-206). The binding of eEF1A present in lysates of MDMs was measured in GST pull-down assays using WT and mutated GST-Nef constructs. Input corresponds to $10 \%$ of the material used for pull-down. Results are representative of two independent experiments

transfected with Exp-t siRNA was accompanied by decreased cytoplasmic eEF1A (Figure 5d). Our results indicate a critical role for Exp-t and eEF1A in resistance to apoptosis in BFAtreated MDMs.
Cytochrome $c$-induced caspase- 9 activation is regulated at multiple levels, and tRNA was reported recently to bind to cytochrome $c$, blocking formation of the apoptosome and the subsequent activation of caspase-9. ${ }^{31}$ Therefore, we tested 
a
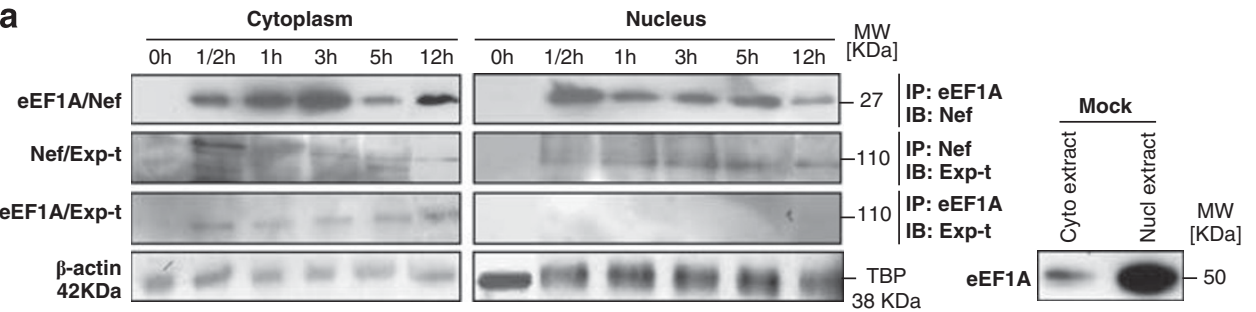

b

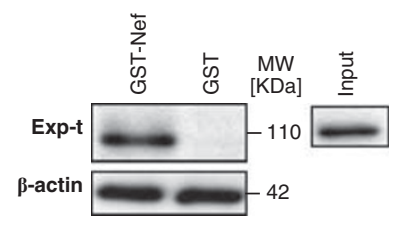

C

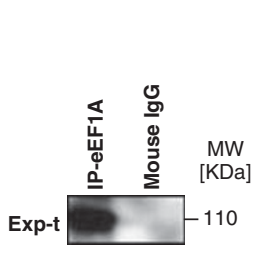

d

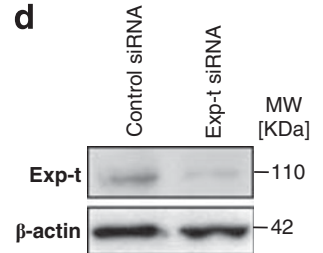

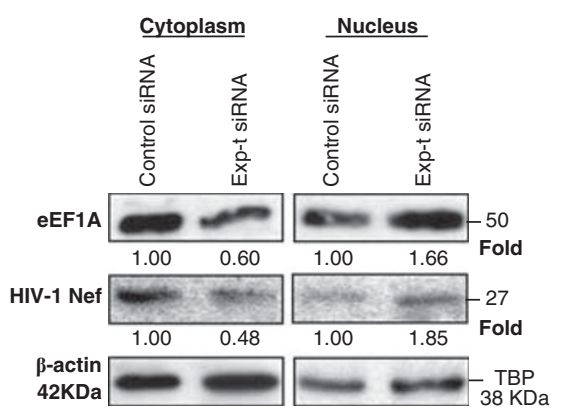

Figure 3 Nuclear-cytoplasmic relocalization of eEF1A/rNef complexes occurs in MDMs treated with rNef and depends on Exp-t. (a) Kinetics of eEF1A/rNef/Exp-t complexes in nuclear and cytoplasmic compartments of MDMs. Nuclear and cytoplasmic extracts of MDMs treated with rNef ( $100 \mathrm{ng} / \mathrm{ml})$ were prepared and the eEF1A/rNef, rNef/Exp-t and eEF1A/Exp-t interactions assessed in both cellular compartments up to $12 \mathrm{~h}$ post-treatment using co-immunoprecipitation and western blotting. Similarly, nuclear and cytoplasmic extracts of untreated MDMs (Mock) were prepared and tested for the expression of eEF-1A. $\beta$-Actin and TBP were used as loading controls. Results are representative of three independent experiments. (b) Using wild-type GST-Nef constructs, the binding of Exp-t present in MDM lysates was assessed in GST pull-down assays. Input corresponds to $10 \%$ of the material used for pull-down. Results are representative of two independent experiments. (c) eEF1A interacts with Exp-t in MDM lysates. Total MDM extracts were prepared and the eEF1A/Exp-t interaction assessed by immunoprecipitation with an anti-eEF1A antibody and western blotting with an anti-Exp-t monoclonal antibody. Results are representative of three independent experiments. (d) Knockdown of Exp-t protein by siRNA in MDMs. MDM cultures were transfected with scrambled control or Exp-t siRNA and total cellular extracts prepared $48 \mathrm{~h}$ post-transfection. Protein expression was analyzed by western blot. $\beta$-Actin was used as a loading control. (e) Effects of Exp-t siRNA on nuclear-cytoplasmic transport of eEF1A and rNef in MDMs. MDM cultures were transfected with a scrambled control or Exp-t siRNA for $48 \mathrm{~h}$ before treatment with $\mathrm{rNef}(100 \mathrm{ng} / \mathrm{ml})$ for $3 \mathrm{~h}$. Nuclear and cytoplasmic extracts were prepared and analyzed by western blot using anti-Nef and anti-eEF1A antibodies. $\beta$-Actin and TBP were used as loading controls. Results are representative of two independent experiments. Protein levels of eEF1A and Nef after siRNA transfection were quantified by densitometry using ImageJ 1.40 software (protein levels in cells transfected with scrambled siRNAs were arbitrarily established at 1)

whether Nef can prevent cytochrome $c$ release in the cytoplasm. We found that most of the cytochrome $c$ was detected in the mitochondrial fraction from normal MDMs, whereas the majority of the cytochrome $c$ in BFA-treated MDMs was detected in the cytosolic fraction (Figure 5e). In Nef-treated MDMs, most of the cytochrome $c$ was detected in the mitochondrial fractions, even after BFA stimulation, which suggests that mitochondrial cytochrome $c$ release is blocked by Nef treatment (Figure $5 \mathrm{e}$ ). The release of mitochondrial cytochrome $c$ into the cytoplasm in BFAstimulated MDMs was inhibited mostly at high Nef concentrations (Figure 5f).

As shown in Figure $5 \mathrm{~g}$, cleavage of $45-\mathrm{kDa}$ procaspase-9 to a $35-\mathrm{kDa}$ fragment in MDMs treated with BFA was blocked by Nef, but not by inactivated boiled Nef, confirming that BFAinduced apoptosis activates caspase- 9 and that it can be inhibited by Nef. The processing of caspase- 9 induced by BFA in Nef-treated MDMs was inhibited in a dose-dependent manner (Figure $5 \mathrm{~h}$ ), indicating that Nef may function by preventing the activation of caspase- 9 via decreased release of mitochondrial cytochrome c. As shown in Figure 5i, pretreatment with $\mathrm{rNef}$ did not inhibit the processing of caspase-3 and caspase-9 induced by TM or TG.
eEF1A/Nef complexes contain tRNAs and block stressinduced apoptosis in MDMs through tRNA binding to cytochrome $\boldsymbol{c}$. As both eEF1A and HIV-1 Nef bind to tRNAs preferentially, ${ }^{32}$ we specifically assessed the presence of several tRNAs in the rNef/eEF1A complexes in MDMs treated with $\mathrm{rNef}$. MDMs treated with rNef were lysed and the presence of tRNAs in the immunoprecipitated eEF1A/ rNef complexes analyzed using RT-PCR. We detected the presence of all tested tRNAs, namely tRNAMet, tRNAPhe, tRNATry and tRNALys in the eEF1A/rNef complexes in MDMs (Figures $6 \mathrm{a}-\mathrm{C}$ ). The $\Delta \Delta C_{\mathrm{t}}$ values were determined separately for untreated cells and averaged around 1 (Supplementary Figure 2).

The binding of tRNAs to cytochrome $c$ was recently reported to inhibit caspase-9 activation. Therefore, we hypothesized that the enhanced nuclear-cytoplasmic transport of eEF1A/rNef/tRNA complexes mediated by Exp-t might result in higher levels of cytosolic tRNAs, which could target cytochrome $c$ released in BFA-treated MDMs. Cytoplasmic extracts of MDMs treated with increasing amounts of $r$ Nef were tested for the presence of tRNAs complexed with immunoprecipitated eEF1A and cytochrome $c$. We observed that tRNA binds to eEF1A in the cytosol of 
BFA-treated MDMs in a Nef-dependent manner (Figure 6d). Similar results were obtained for the binding of tRNA to cytochrome $c$ (Figure $6 \mathrm{e}$ ), indicating that Nef treatment of BFA-stimulated MDMs favors the formation of tRNA/ cytochrome $c$ complexes in the cytoplasm. Cytoplasmic extracts of BFA-stimulated MDMs treated with rNef, in the absence or presence of Exp-t siRNA, Exp-1 siRNA or Exp-5 siRNA, were analyzed for the presence of tRNA binding cytochrome $c$. Knockdown of Exp-t, but not Exp-1 or Exp-5, decreased the amount of tRNA bound to cytochrome $c$, a
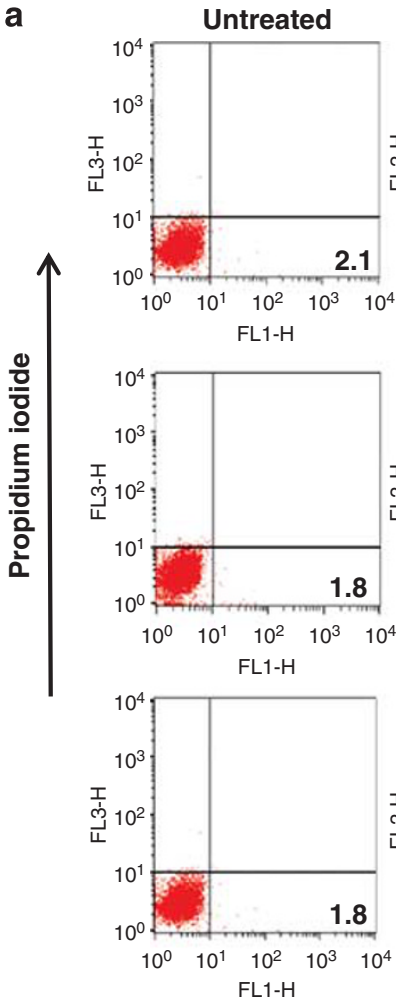

BFA 12h
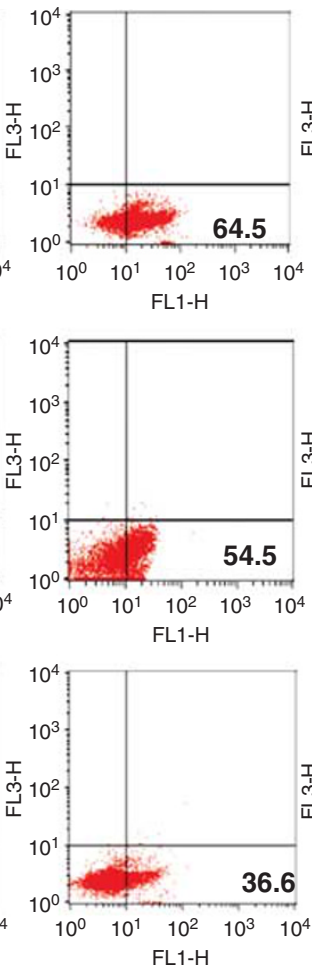

BFA 15h

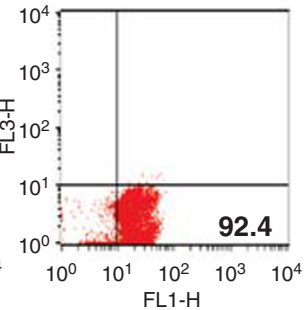

Mock

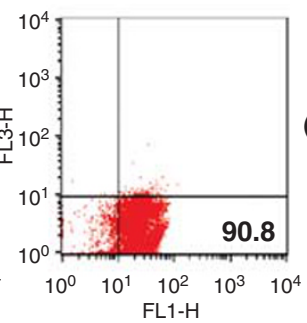

Nef

$(0.125 \mathrm{ug} / \mathrm{ml})$

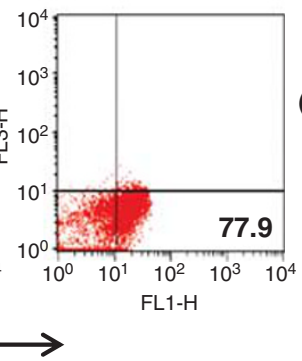

Nef (0.750ug/ml)

Annexin V

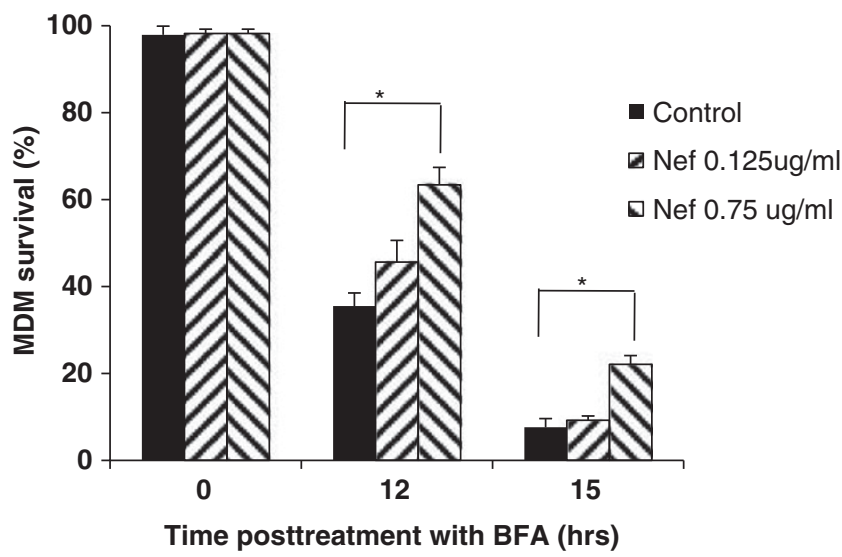

Figure 4 rNef-mediated inhibition of BFA-induced apoptosis in MDMs parallels cytoplasmic accumulation of eEF1A and is dependent on Exp-t. (a) rNef prevents BFAinduced apoptosis in MDMs. MDMs were treated with BFA $(10 \mu \mathrm{g} / \mathrm{ml})$ for 12 or $15 \mathrm{~h}$ or untreated in the presence of increasing concentrations of $\mathrm{rNef}(0,125,750 \mathrm{ng} / \mathrm{ml})$. Apoptosis was detected by flow cytometric analysis of annexin-V. The histogram summarizes the survival of MDMs following treatment with BFA ( $10 \mu \mathrm{g} / \mathrm{ml})$ for 12 or $15 \mathrm{~h}$ in the presence of increasing concentrations of rNef. The results represent means of three independent experiments; ${ }^{*} P<0.05$. (b) rNef prevents BFA-induced apoptosis, but neither TM-induced apoptosis nor TG-induced apoptosis in MDMs. MDMs were treated with BFA (10 $\mu \mathrm{g} / \mathrm{ml})$, TM $(10 \mu \mathrm{g} / \mathrm{ml})$, or TG $(10 \mu \mathrm{g} / \mathrm{ml})$ for $5 \mathrm{~h}$ or $12 \mathrm{~h}$ or untreated in the presence of $\mathrm{rNef}(1 \mu \mathrm{g} / \mathrm{ml})$. Apoptosis was measured by TUNEL assay. Results are representative of data observed in three independent experiments. (c) The C-terminal extremity of Nef prevents BFA-induced apoptosis in MDMs. MDMs were treated with BFA (10 $\mu \mathrm{g} / \mathrm{ml})$ for $12 \mathrm{~h}$ or untreated in the presence of WTNef, Nef1-60, or Nef55-206 $(1 \mu \mathrm{g} / \mathrm{ml})$. Apoptosis was measured by TUNEL assay. Results are representative of data observed in two independent experiments. (d) Blockade of BFA-induced apoptosis in MDMs by rNef is dependent on Exp-t. MDM cultures were transfected with a scrambled control, Exp-t siRNA, Exp-1 siRNA or Exp-5 siRNA for $48 \mathrm{~h}$ before treatment with BFA $(10 \mu \mathrm{g} / \mathrm{ml})$ for $12 \mathrm{~h}$ or untreated, in the presence $(1 \mu \mathrm{g} / \mathrm{ml})$ or absence of $r$ Nef. Apoptosis was detected by flow cytometric analysis of annexin-V. The histogram shows the survival percentage of MDMs treated with mock, BFA or BFA + Nef in the presence of exportin siRNAs. Results are representative of three independent experiments; ${ }^{*} P<0.05$ 

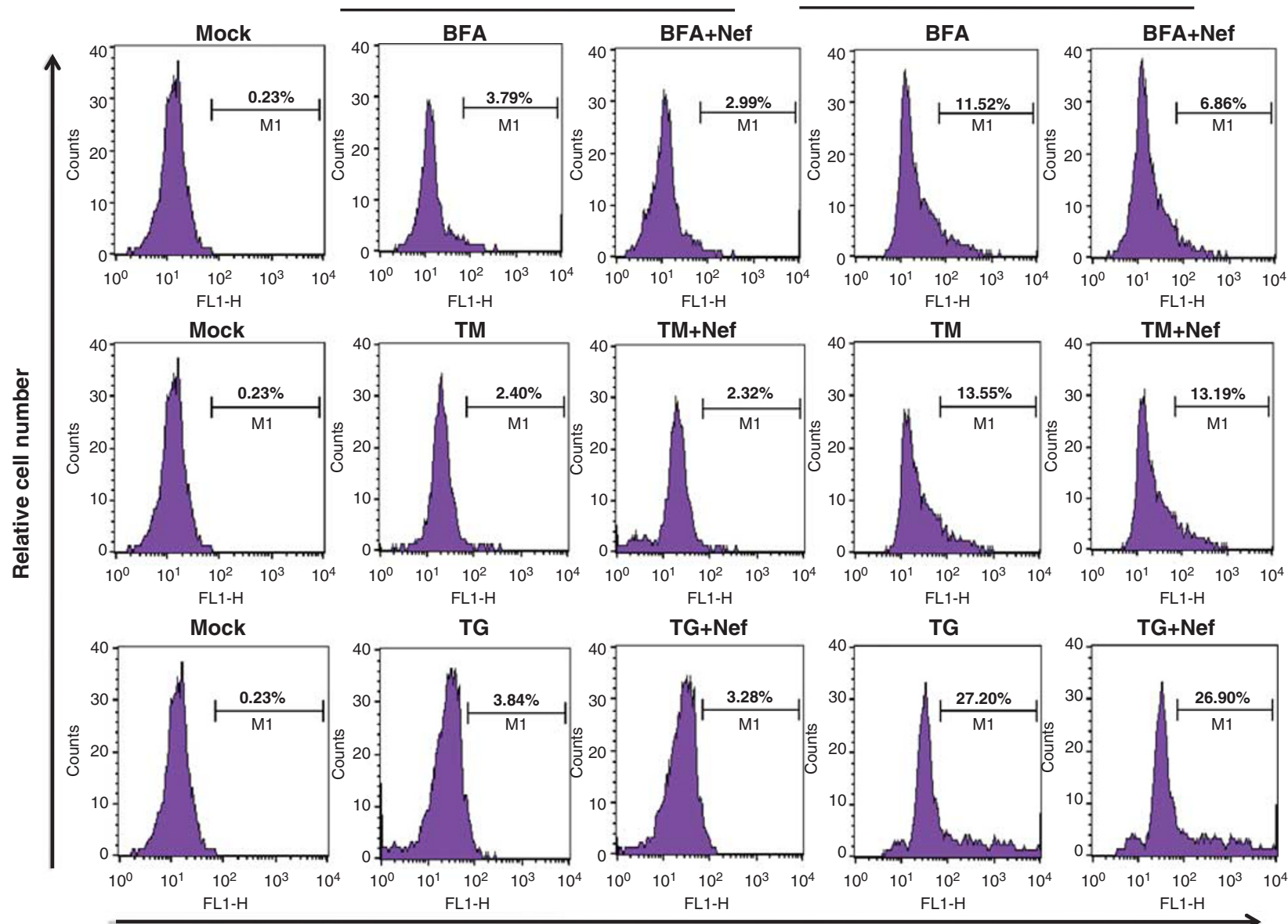

TdT Labeling (log fluorescence)

C
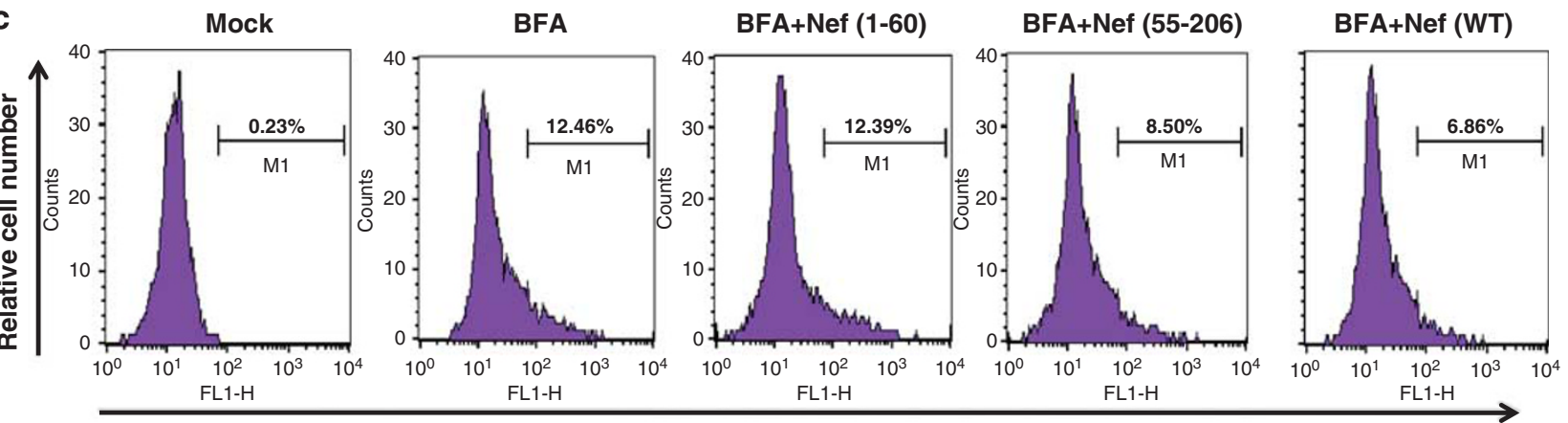

TdT Labeling (log fluorescence)

Figure 4 Continued

as well as eEF1A, in BFA-stimulated MDMs treated with Nef (Figures $6 f$ and $g$ ).

\section{Discussion}

We observed a direct interaction between HIV-1 Nef and eEF1A. Nuclear-cytoplasmic relocalization of rNef/eEF1A/ tRNA complexes prevented stress-induced apoptosis of MDMs treated with BFA through decreased release of mitochondrial cytochrome $c$, enhanced tRNA binding to released cytosolic cytochrome $c$ and increased cytoplasmic levels of eEF1A, ultimately resulting in decreased caspase activation (Figure 7). Our results indicate that the HIV-1 Nef protein enhances resistance to stress-induced apoptosis in MDMs through the nuclear-cytoplasmic relocalization of rNef/ eEF1A/tRNA complexes.

Our results show that nuclear-cytoplasmic transport of eEF1A/Exp-t/tRNA complexes occurs in MDMs treated with rNef. Although the role of eEF1A in tRNA transport has been reported, this is the first time that a viral protein such as Nef 

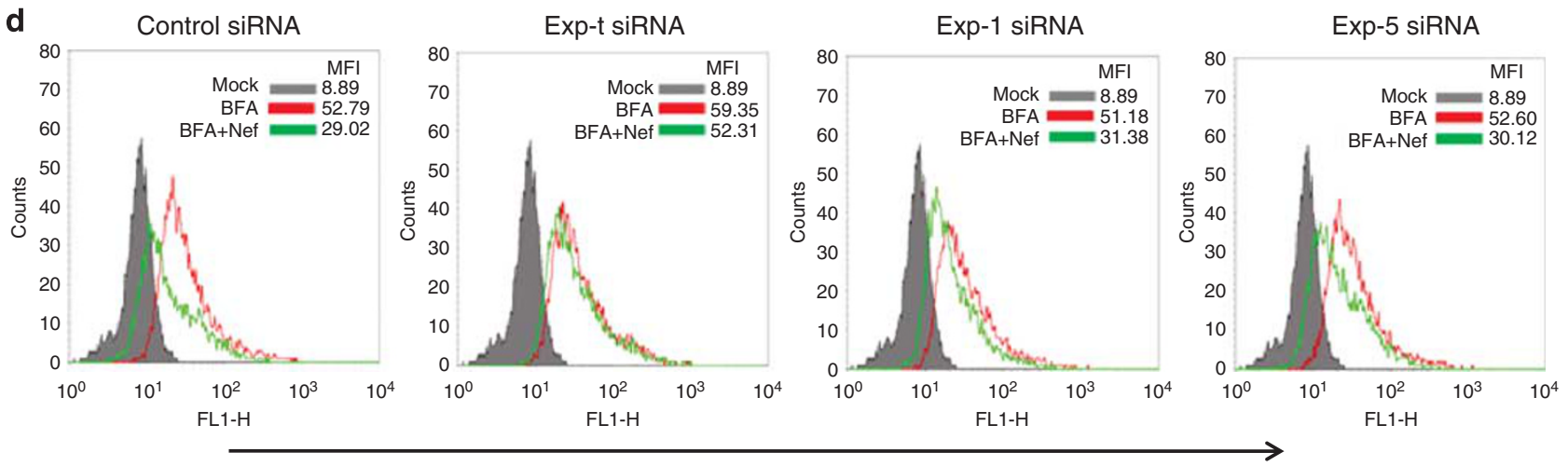

Annexin V

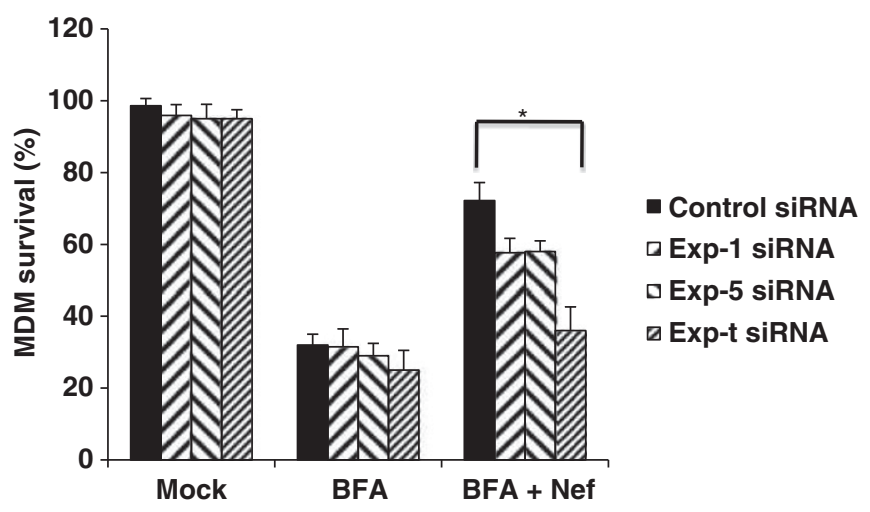

Figure 4 Continued

has been shown to be actively involved in such a process. The presence of large amounts of eEF1A in the nucleus of MDMs confirms previous results indicating that eEF1A is localized in both the cytoplasm and nucleus of cells. We also observed that the nuclear-cytoplasmic distribution of eEF1A/rNef complexes included the presence of RNA (Figures $6 a-c$ ). Both proteins most probably bind to each other in a RNAindependent manner. First, we observed the interaction between Nef and eEF1A in a purified system (Figure 2a). Second, supportive experimental evidence comes from crosslinking studies between rabbit EF-1 $\alpha$.GTP and aminoacyl-tRNA that indicate contact sites corresponding to residues 270-275, 345-355 and 375, sites that are in contact with the RNA in the ternary complex crystal. ${ }^{33}$ Finally, the N-terminal Arg-rich region of $\mathrm{HIV}-1 \mathrm{Nef}$ (aa 1-22) binds to RNA in vitro. ${ }^{32}$ As the Nef fragment encoding amino acids 55 to 206 is sufficient for binding to the N-terminal extremity of eEF1A (Figure 2b), it is highly unlikely that the Nef interaction with eEF1A requires the presence of RNA.

We hypothesized that tRNA is the most likely candidate to be present in the Nef-eEF1A complex because eEF1A binds to aminoacyl-tRNA during translation and Nef has been shown to bind to tRNAs and promote the annealing of primer tRNA to viral genomic RNA. In addition, Exp-t, rather than Exp-5, has a critical role in this process, which is in agreement with a critical role of Exp-t in the selection and transport of mature tRNAs from the nucleus to the cytoplasm.

Nef has been reported to prevent both Fas and TNF receptor-mediated deaths in HIV-infected T cells via interaction with ASK-1, thereby blocking the extrinsic apoptosis pathway. Nef inhibits ASK-1, caspase-3 and caspase-8 activation, resulting in the blockade of apoptosis in HIVinfected cells. Nef has also been shown to block the intrinsic apoptosis pathway via the inhibition of the proapoptotic protein Bad. ${ }^{34}$ Our data highlight a new molecular mechanism elicited by the HIV-1 Nef protein to block the intrinsic apoptosis pathway in Nef-expressing cells, such as HIV-1-infected MDMs. The HIV-1 Nef protein, through the nuclear-cytoplasmic relocalization of $\mathrm{rNef} / \mathrm{eEF} 1 \mathrm{~A} / \mathrm{tRNA}$ complexes, could participate in preventing stress-mediated effects at several levels. First, the decreased cytochrome $c$ release is probably due to cytoplasmic accumulation of eEF1A that stabilizes actin filaments and potentially could block the BFA-induced disruption of the organization of the microtubule and actin cytoskeleton. Second, the tRNAs could be specifically delivered by the eEF1A/Nef/tRNA complexes to cytosolic cytochrome $c$, thereby preventing cytochrome $c$ interaction with Apaf-1 and blocking caspase activation and apoptosis. Third, the eEF1A protein could be delivered by the eEF1A/ Nef/tRNA complexes in the cytoplasm to participate in cytoskeleton rearrangement aimed to eliminate unfolded proteins produced during stress events. ${ }^{35-37}$ Finally, eEF1A has been reported to activate Akt, the protein kinase $B$ reported by numerous studies to favor resistance to apoptosis. ${ }^{11}$ In order to determine the scope of eEF1A-mediated protection, we assayed eEF1A antiapoptotic activity in response to ER-stress agents, BFA, TM and TG. BFA reversibly blocks protein transport between the ER and Golgi, whereas TM blocks the synthesis of all $\mathrm{N}$-linked glycoproteins and TG inhibits the ER $\mathrm{Ca}^{2+}$-ATPase family of calcium pumps. 
a

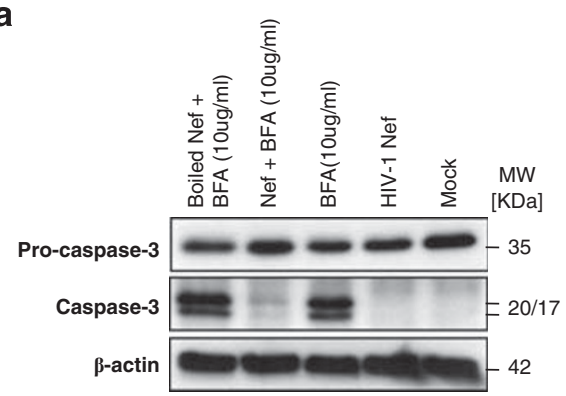

C

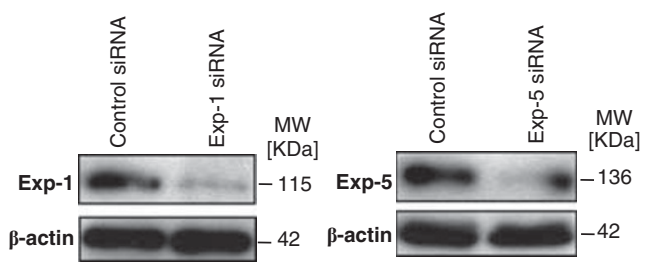

b

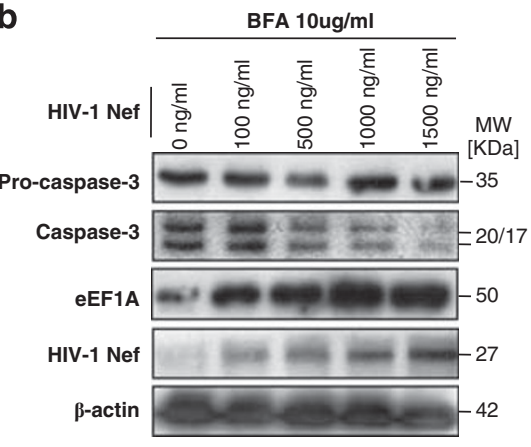

d

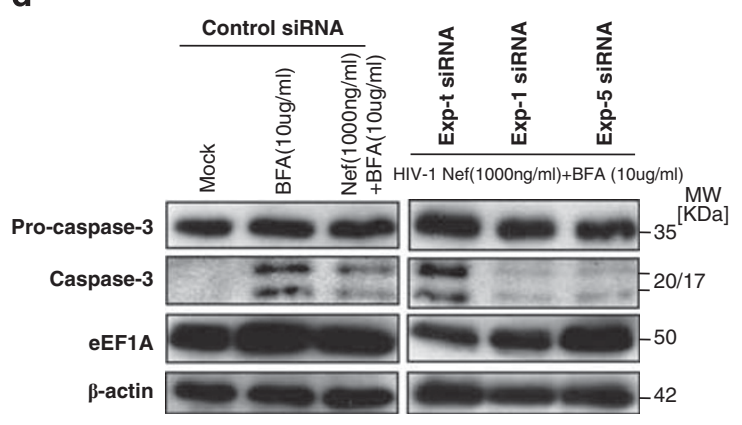

g

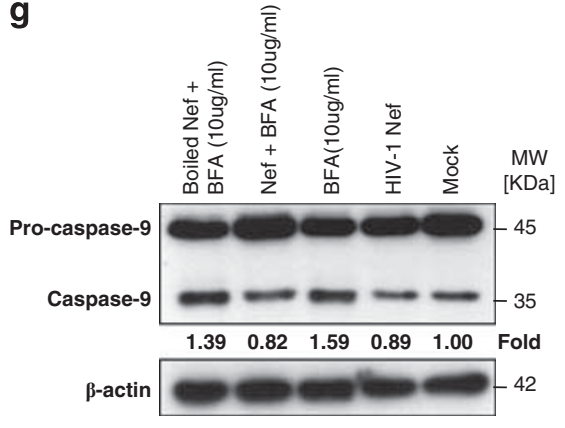

h

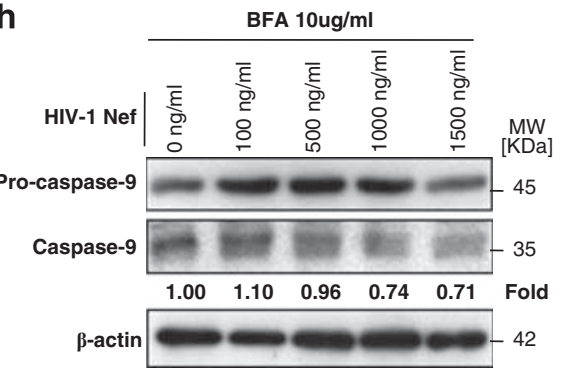

Figure 5 rNef-mediated cytoplasmic accumulation of eEF1A in BFA-treated MDMs inhibits caspase activation and decreases the cytoplasmic release of cytochrome $c$. (a) Inhibition of caspase-3 activation in BFA-stimulated MDMs treated with $\mathrm{rNef}(1000 \mathrm{ng} / \mathrm{ml})$. (b) Inhibition of caspase-3 activation in BFA-stimulated MDMs treated with rNef is dose-dependent and positively correlates with cytoplasmic accumulation of eEF1A. (c) Knockdown of Exp-1 and Exp-5 proteins by siRNA in MDMs. MDM cultures were transfected with scrambled control, Exp-1 siRNA or Exp-5 siRNA. Total cellular extracts were prepared $48 \mathrm{~h}$ post-transfection. Protein expression was analyzed by western blot. $\beta$-Actin was used as a loading control. (d) Inhibition of caspase-3 activation in BFA-stimulated MDMs treated with rNef is dependent on Exp-t. (e) Mitochondrial cytochrome $c$ release in BFA-treated MDMs is blocked by rNef. (f) Mitochondrial cytochrome $c$ release in BFA-treated MDMs is blocked by rNef in a dose-dependent manner and positively correlates with cytoplasmic accumulation of eEF1A. (g) Inhibition of caspase-9 activation in BFA-stimulated MDMs treated with rNef (1000 ng/ml). Protein levels of caspase-9 were quantified by densitometry using ImageJ 1.40 software (the level of caspase-9 in mock cells was arbitrarily established at 1). (h) Inhibition of caspase-9 activation in BFA-stimulated MDMs treated with rNef is dose-dependent. Protein levels of caspase- 9 were quantified by densitometry using ImageJ 1.40 software (the level of caspase-9 in mock cells was arbitrarily established at 1). (i) Inhibition of caspase activation in BFA-stimulated MDMs treated with rNef, but neither in TM-stimulated MDMs nor in TG-stimulated MDMs treated with rNef. Left panel, Time course of caspase activation in BFA-stimulated MDMs, TM-stimulated MDMs, or TG-stimulated MDMs. Right panel, Inhibition of caspase activation in BFA-stimulated MDMs treated with rNef, but neither in TM-stimulated MDMs nor in TG-stimulated MDMs treated with rNef. MDM cultures were treated with BFA $(10 \mu \mathrm{g} / \mathrm{ml})$, TM $(10 \mu \mathrm{g} / \mathrm{ml})$ or TG $(10 \mu \mathrm{g} / \mathrm{ml})$ for $12 \mathrm{~h}$ in the presence of $\mathrm{rNef}(1 \mu \mathrm{g} / \mathrm{ml})$, and caspase-3 and caspase-9 activation was measured in total cellular lysates. Results are representative of data obtained in three independent experiments 
i
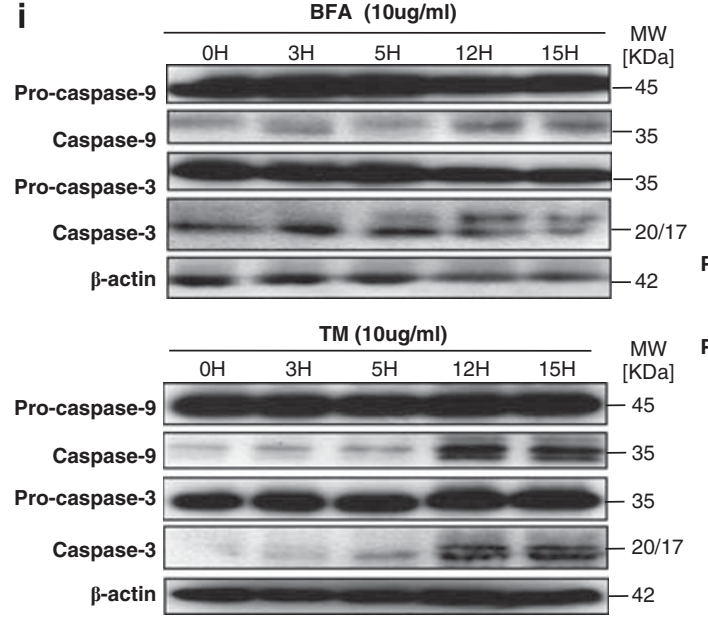

$\mathrm{TG}(10 \mathrm{ug} / \mathrm{ml})$

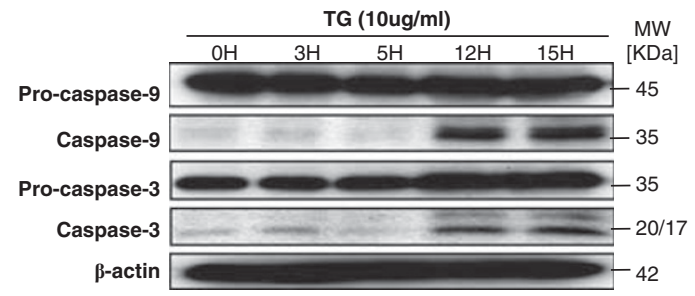

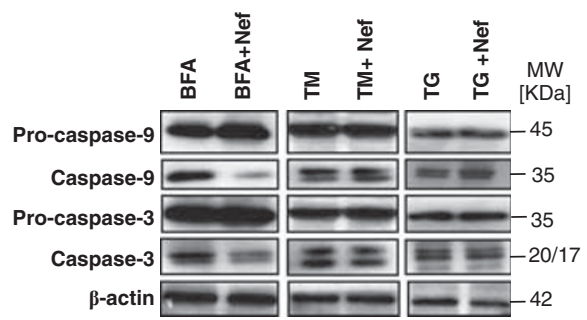

Figure 5 Continued

Our results indicate that Nef specifically blocks apoptosis induced by the ER-stress agent BFA, indicating that increased cytoplasmic relocalization of eEF1A by HIV-1 Nef may alleviate the inhibition of protein transport between the ER and Golgi observed in BFA-treated cells. In addition, Nef could favor an homeostatic control of UPR and of oxidative stress observed during the acute phase of HIV infection and subsequently fuel the progression of the disease toward a chronic infection with the formation of cellular reservoirs of the virus.

Taken together, the results indicate that cytoplasmic relocalization of the eEF1A/tRNAs complexes by HIV-1 Nef could ultimately favor the survival of the cell under stress, thereby allowing optimal viral replication. This event may participate in the formation and maintenance of viral reservoirs in HIV-infected persons.

\section{Materials and Methods}

Cell culture. Primary human MDMs were prepared from the peripheral blood of healthy donors and cultured in RPMI medium supplemented with $10 \%$ (V/v) pooled AB human serum (Sigma, Munich, Germany) as described previously. ${ }^{38}$ PBLs were cultivated in RPMI medium supplemented with $10 \%$ (v/v) fetal bovine serum (FBS). Vero cells, MRC5 cells and promonocytic U937 cells were obtained from the American Tissue Cell Culture Collection (ATCC, Manassas, VA, USA). The cell lines were cultivated in RPMl 1640 supplemented with 10\% FBS, $1 \%$ glutamine and $1 \%$ penicillin/streptomycin.

Recombinant Nef treatment. MDMs $\left(5 \times 10^{6}\right.$ cells $)$ were treated with recombinant myristoylated Nef protein (rNef) from Jena Bioscience (Jena, Germany). Cell pellets were collected at various times after treatment, washed extensively and either lysed for western blot analysis or fixed with $3 \%$ paraformaldehyde in PBS for $30 \mathrm{~min}$ for flow cytometric analysis. The bacterially expressed GST-Nef(WT), GST$\operatorname{Nef}(1-60)$ and GST-Nef(55-206) were purified and cleaved with thrombin (cat. no. 27-0846-01; GE Healthcare, Little Chalfont, Buckinghamshire, UK) according to the instructions provided by the manufacturer. SDS-Tricine polyacrylamide gel electrophoresis (PAGE) and Coomassie staining was done to visualize the thrombin cleaved products of HIV-1 Nef wild-type and mutants.
Isolation of nuclear and cytoplasmic extracts. Nuclear and cytoplasmic extracts were isolated as described previously. ${ }^{38}$ Protein concentrations in the nuclear and cytoplasmic extracts were determined by the Bradford method using a BioPhotometer (Eppendorf, Hamburg, Germany).

Preparation of cytosolic and mitochondrial fractions. Cytosolic and mitochondrial fractions were prepared using the cell fractionation kit-standard (\#MS861, Mitosciences, Eugene, OR, USA). The coxIV (Cell Signaling Technology, Danvers, MA, USA) antibody was used as a mitochondrial loading control.

Immunoprecipitation. Vero cells, MRC5 cells, U937 cells, PBLs or primary MDMs were left untreated or treated with rNef for different periods of time. Cell lysates were precleared by adding $50 \mu \mathrm{l}$ of Protein-G Plus/Protein A-Agarose (Calbiochem-Novabiochem, Bad Soden, Germany) for $1 \mathrm{~h}$ at $4{ }^{\circ} \mathrm{C}$. The cleared supernatants were removed, combined with $5 \mu \mathrm{g}$ anti-Nef or anti-EF1A antibodies (Chemicon, Upstate, Billerica, MA, USA), and incubated overnight at $4{ }^{\circ} \mathrm{C}$. Immune complexes were washed in the presence of protease inhibitors, the bound proteins eluted with sample buffer and the proteins run on a $10 \%$ SDSPAGE gel. SDS-PAGE and western blot analysis were performed according to standard procedures. $\beta$-Actin and TATA binding protein (TBP; Sigma, St. Louis, MO, USA) were used as cytoplasmic or nuclear loading controls, respectively. Western blots were developed using the ECL detection kit (Amersham Pharmacia Biotech, Little Chalfont, Buckinghamshire, UK).

Far-western identification of Nef protein interactions. Proteinprotein interaction screening was performed on $h E \times 1$ high-density protein expression cDNA libraries with rNef protein, which was expressed by the prokaryotic expression vector pET-32 (Novagen, Madison, WI, USA) according to previously published protocols.

Plasmids and construction of expression vectors. The pcDNA6based expression vectors for HIV-1 Nef and eEF1A have been described elsewhere. ${ }^{39}$ Deletion constructs were generated by standard PCR and cloning procedures. HIV-1 Nef and EF1A constructs were verified by DNA sequencing. eEF1A/Nef interactions were detected in vivo by transient co-transfection of GAL4-Nef and VP16-eEF1A in the context of a mammalian two-hybrid system (CheckMate, Promega, Madison, WI, USA). Sequence authenticity was confirmed by cDNA cycle sequencing. 
a

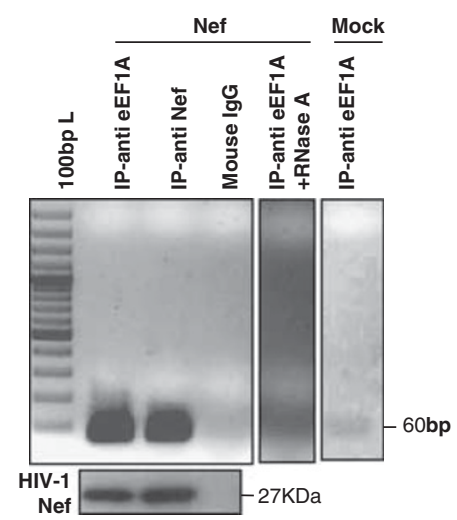

b

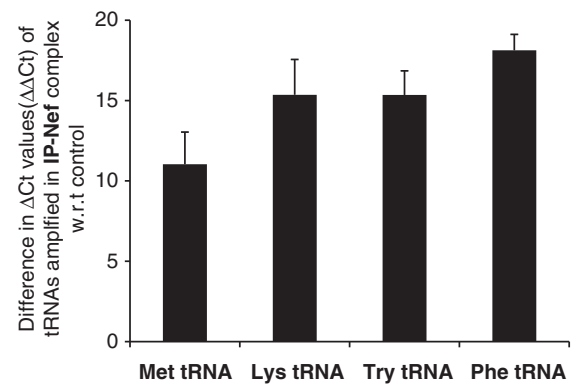

e
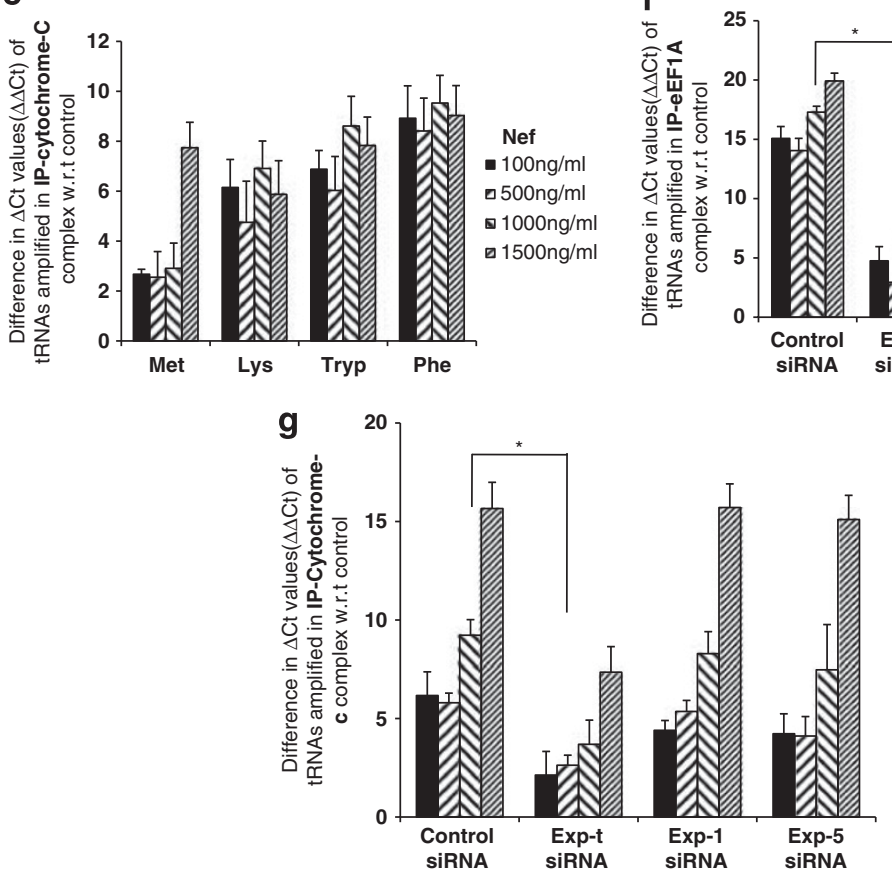

c

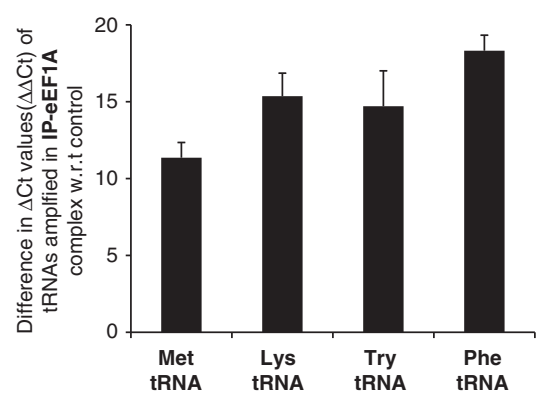

d
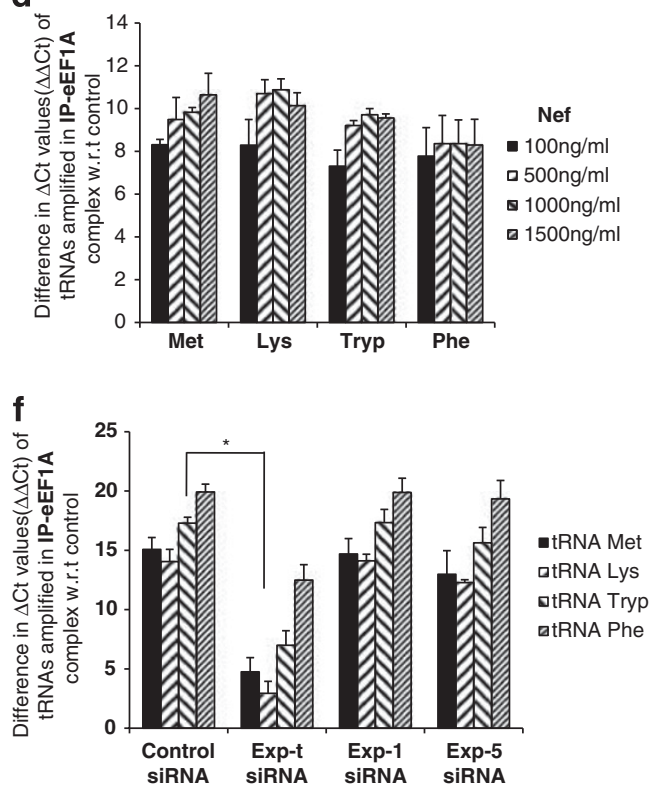

- tRNA Met

口 tRNA Lys

- tRNA Tryp

tRNA Phe

Figure 6 rNef-mediated cytoplasmic accumulation of eEF1A in BFA-treated MDMs parallels tRNA binding to cytochrome $c$. (a) Direct detection of tRNAs in rNef/eEF1A complexes in MDMs treated with $\mathrm{rNef}$. Lysates from MDMs treated with $\mathrm{rNef}(100 \mathrm{ng} / \mathrm{ml})$ for $3 \mathrm{~h}$ or left untreated (Mock) were prepared, immunoprecipitated with anti-eEF1A and anti-Nef antibodies, and tRNALys in the eEF1A/rNef complexes amplified by RT-PCR. Lysates were also immunoprecipitated with isotype control antibody. The presence of HIV-1 Nef protein in the immune complex was determined by western blot. As an internal control, lysates from MDMs treated with $\mathrm{rNef}(100 \mathrm{ng} / \mathrm{ml})$ for $3 \mathrm{~h}$ were prepared, treated with RNAse A $(10 \mu \mathrm{g} / \mathrm{ml})$ for $30 \mathrm{~min}$, immunoprecipitated with an anti-eEF1A antibody, and tRNALys in the eEF1A/Nef complexes was amplified by RT-PCR. (b and $\mathbf{c})$ MDMs were treated with $\mathrm{rNef}(100 \mathrm{ng} / \mathrm{ml})$; the lysates were immunoprecipitated with an anti-Nef $\mathrm{Ab}(\mathbf{b})$ or anti-eEF1A Ab (c) and the presence of tRNAMet, tRNALys, tRNATry and tRNAPhe in the Nef/eEF1A complex determined by qRT-PCR. (d and e) MDMs were treated with BFA for $12 \mathrm{~h}$ in the presence or absence of rNef $(0-1500 \mathrm{ng} / \mathrm{ml})$; the lysates were immunoprecipitated with an anti-eEF1A Ab (d) or anti-cytochrome $c A b(\mathbf{e})$, and the presence of tRNAMet, tRNALys, tRNATry, and tRNAPhe was determined by qRT-PCR. (f and $\mathbf{g}$ ) The presence of tRNAs associated with eEF1A and cytochrome $c$ in BFA-stimulated MDMs treated with rNef is dependent on Exp-t. MDM cultures were transfected with scrambled control siRNA, Exp-t siRNA, Exp-1 siRNA or Exp-5 siRNA. Cytoplasmic extracts of MDMs treated with BFA (10 $\mu \mathrm{g} / \mathrm{ml})$ for $12 \mathrm{~h}$ in the absence or presence of rNef $(1000 \mathrm{ng} / \mathrm{ml})$ were prepared $48 \mathrm{~h}$ post-transfection, immunoprecipitated with an anti-eEF1A Ab (f) or anti-cytochrome $c \mathrm{Ab}(\mathbf{g})$, and the presence of tRNALys, tRNAMet, tRNAPhe, and tRNATry binding to eEF1A and cytochrome $c$ was determined using qRT-PCR. Results representative of three independent experiments are shown; ${ }^{*} P<0.05$ 


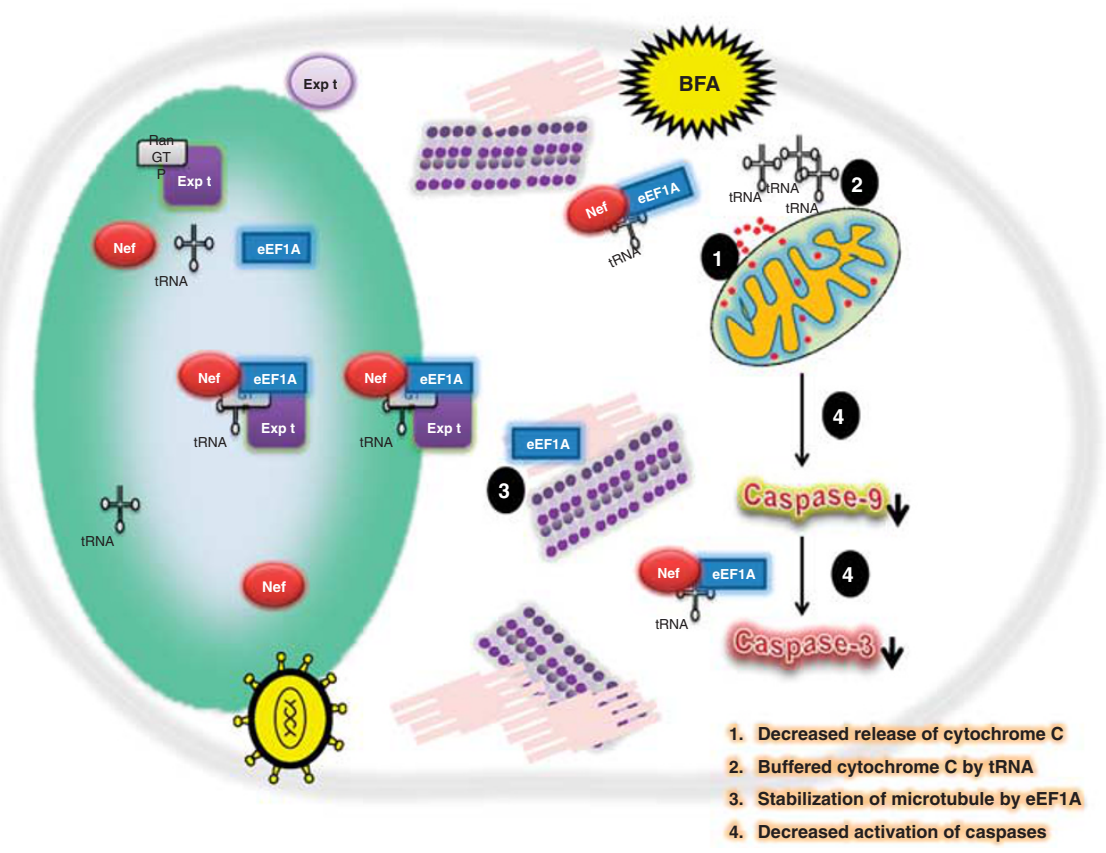

Figure 7 Potential inhibitory effect of the eEF1A/rNef/tRNA complex on the intrinsic apoptotic pathway in BFA-treated MDMs. HIV-1 Nef protein favors translocation and accumulation of eEF1A from nucleus toward to the cytoplasm of the cell. tRNA is present in the Nef/eEF1A complex, which is exported by exportin-t and buffers the cytochrome $c$ released under oxidative stress conditions. At the same time the Nef/eEF1A complex inhibits the caspase activation. Further, eEF1A could stabilize the microtubules and give relief to the cell to survive under stress conditions. BFA, brefeldin-A; Expt, exportin-t

HIV-1 Nef wild type, $\mathrm{N}$ terminus (1-60) or $\mathrm{C}$ terminus (55-206) was cloned into pGEX-4T-1 expression vector. For protein purification purposes, each construct was transformed into bacteria (BL21DE from Novagen). A single colony was inoculated in $5 \mathrm{ml} \mathrm{LB}$ broth plus $50 \mathrm{mg} / \mathrm{ml}$ ampicillin at $37^{\circ} \mathrm{C}$ in a shaker overnight. A $1 \mathrm{ml}$ aliquot of the overnight bacterial culture was used to inoculate $300 \mathrm{ml} \mathrm{LB}$ broth plus $50 \mathrm{mg} / \mathrm{ml}$ ampicillin and grown for $2.5 \mathrm{~h}$ at $37^{\circ} \mathrm{C}$ in a shaker (until $\mathrm{OD}_{600}=0.5$ ). IPTG was added at a final concentration of $0.1 \mathrm{mM}$ and the culture grown for an additional $3 \mathrm{~h}$ at $37^{\circ} \mathrm{C}$ in the shaker. Bacteria were pelleted, washed with ice-cold TBS, and lysed in bacterial protein extraction reagents (Pierce, Rockford, IL, USA). eEF1A and mutants were constructed and expressed in the bacteria as described previously. ${ }^{25}$

Transfection assay. To carry out transient transfections, the DNA concentration was kept constant in the different samples by using the corresponding empty vector. A total of $5 \times 10^{6}$ cells were transfected with 5-10 $\mu \mathrm{g}$ total plasmid DNA, which included the firefly luciferase-expressing vector using the DEAE-dextran procedure. Luciferase activity in cell lysates was measured $48 \mathrm{~h}$ post-transfection using a luminometer (TD-20/20, Promega). Luciferase expression was normalized with respect to protein concentration using the Detergent-Compatible Protein Assay (Bio-Rad, Munich, Germany). All transfections were performed in triplicate.

Pull-down assays. Fragments of the cDNA encoding HIV-1 $1_{89.6}$ Nef were produced using convenient restriction enzymes and PCR methods and then cloned into the GST fusion vector pGEX-4T-1 (Amersham Biosciences, Little Chalfont, Buckinghamshire, UK). The GST constructs were transformed into the E. coli strain BL21 (Stratagene, La Jolla, CA, USA), and the GST fusion proteins were purified according to the manufacturer's instructions. For pull-down assays, $20 \mu \mathrm{g}$ of the GST-Nef proteins (NefWT, Nef(1-60), Nef(55-206)) kindly provided by Dr. Fackler (University of Heidelberg, Germany) were incubated overnight at $4{ }^{\circ} \mathrm{C}$ with $1500 \mu \mathrm{g}$ of MDM lysate. The suspension was then washed three times in PBS, denaturized and subsequently separated by SDS-PAGE before autoradiography.

RNA interference. MDM cultures $\left(0.5 \times 10^{6}\right.$ cells $)$ were transfected with a scrambled control, Exp-t siRNA, Exp-1 siRNA or Exp-5 siRNA duplex (Santa-Cruz Biotechnology, Santa Cruz, CA, USA) using Lipofectamine RNAiMAX (Invitrogen, Carlsbad, CA, USA). MDMs were treated with rNef $48 \mathrm{~h}$ post-transfection, and
eEF1A, rNef, Exp-t, Exp-1 and Exp-5 protein expression and MDM apoptosis analyzed by western blot and annexin-V (Invitrogen) assay, respectively. Transfection efficiency was monitored using a fluorescein-conjugated scrambled control duplex, exceeding $80 \%$ in MDMs.

tRNA interaction with cytochrome $c$, eEF1A or Nef. To examine the tRNA/cytochrome $c$ interaction in vivo, MDMs transfected with Exp-t siRNA, Exp-1 siRNA, Exp-5 siRNA, or scramble siRNA for $48 \mathrm{~h}$ before treatment with $\mathrm{rNef}$ were harvested and washed twice with ice-cold PBS. The cytoplasmic extracts were immunoprecipitated with anti-cytochrome $c$ antibody (Santa-Cruz Biotechnology) at $4{ }^{\circ} \mathrm{C}$ overnight followed by incubation with protein-G agarose beads for an additional $2 \mathrm{~h}$. The beads were washed and resuspended in $200 \mu \mathrm{l}$ in RNA elusion buffer ( $50 \mathrm{mM}$ Tris, $100 \mathrm{mM} \mathrm{NaCl}, 10 \mathrm{mM}$ EDTA, $0.1 \% \mathrm{SDS}$ ) at $65^{\circ} \mathrm{C}$ for $10 \mathrm{~min}$. RNA was extracted (RNeasy kit, Qiagen, Hilden, Germany) and cDNA synthesized using a cDNA synthesis kit (Invitrogen).

The sequence of the primers (Eurogentec, Liège, Belgium) used in RT-PCR amplification were as follows:

tRNA Met forward

tRNA Met reverse

tRNA Tryp forward

tRNA Tryp reverse

tRNA Phe forward

tRNA Phe reverse

tRNA Lys forward

tRNA Lys reverse

$$
\begin{aligned}
& \text { 5'-CTGGGCCCATAACCCAGAG-3' } \\
& \text { 5'-TAGCAGAGGATGGTTTCGAT-3' } \\
& \text { 5'-GGCTCGTTGGTCTAGGGGTA-3' } \\
& \text { 5'-GATTTGAACCCGGGACCT-3' } \\
& \text { 5'-CCTCCTCAAAGCAATACACTGA-3' } \\
& \text { 5'-GGTGATGTGAGCCCGTCTAA-3' } \\
& \text { 5'-ATAGCTCAGTCGGTAGAGCATCA-3' } \\
& \text { 5'-ACAGGGACTTGAACCCTGGAC-3' }
\end{aligned}
$$

The tRNAs associated with EEF1A and rNef were detected after immunoprecipitation with an anti-eEF1A or anti-Nef antibody, followed by RT-PCR amplification as described above. $C_{t}$ values were analyzed using the $\Delta \Delta C_{t}$ method. ${ }^{40} \Delta C_{t}$ was calculated by normalization to the isotype control, and $\Delta \Delta C_{\mathrm{t}}$ values were calculated by normalizing to the $C_{\mathrm{t}}$ values of the untreated sample. The $\Delta \Delta C_{\mathrm{t}}$ values were also determined separately for untreated cells and averaged around 1 (Supplementary Figure 2).

Detection of apoptosis. The detection of apoptosis by annexin-V-propidium iodide assay (Invitrogen) and TUNEL assay (AbD Serotec, Oxford, UK) was performed as previously described. ${ }^{29}$ For ER-stress agents, an appropriate dose was selected from a range of concentrations tested. BFA (Sigma, USA), TG (Sigma, USA) and TM (Sigma, USA) were used at $10 \mu \mathrm{g} / \mathrm{ml}$. 
Caspase activity. Caspase- 9 and caspase- 3 activity was measured by western blotting as described previously. ${ }^{31}$ Where specified, protein levels were quantified by densitometry using ImageJ 1.40 software (National Institute of Health, Bethesda, MD, USA).

Statistical analysis. Statistical analyses were performed using the analysis of variance test, and differences were considered significant at a value of ${ }^{*} P<0.05$, ${ }^{* *} P<0.01$ and ${ }^{* *} P<0.001$. Microsoft Excel was used to construct the plots.

\section{Conflict of Interest}

The authors declare no conflict of interest.

Acknowledgements. This work was supported by grants from the University of Franche-Comté to $\mathrm{GH}$, and WA and KAK are recipients of doctoral scholarships from the Higher Education Commission, Pakistan.

1. Walter $P$, Ron $D$. The unfolded protein response: from stress pathway to homeostatic regulation. Science 2011; 334: 1081-1086.

2. Shore GC, Papa FR, Oakes SA. Signaling cell death from the endoplasmic reticulum stress response. Curr Opin Cell Biol 2011; 23: 143-149.

3. Elbim C, Pillet S, Prevost MH, Preira A, Girard PM, Rogine N et al. Redox and activation status of monocytes from human immunodeficiency virus-infected patients: relationship with viral load. J Virol 1999; 73: 4561-4566.

4. Masutani $\mathrm{H}$, Ueda S, Yodoi J. The thioredoxin system in retroviral infection and apoptosis. Cell Death Diff 2005; 12: 991-998.

5. Uhm HD, Orenstein JM, Wahl SM. Fas mediates apoptosis in human monocytes by a reactive oxygen intermediate dependent pathway. J Immunol 1996; 156: 3469-3477.

6. Aillet $F$, Masutani $H$, Elbim $C$, Raoul $H$, Chene L, Nugeyre MT et al. Human immunodeficiency virus induces a dual regulation of $\mathrm{Bcl}-2$, resulting in persistent infection of CD4 + T- or monocytic cell lines. J Virol 1998; 72: 9698-9705.

7. Tiedde LM, Cook EA, Morsey B, Fox HS. Oxygen matters: tissue culture oxygen levels affect mitochondrial function and structure as well as responses to HIV viroproteins. Cell Death Dis 2011; 2: e246.

8. Kohler A, Hurt E. Exporting RNA from the nucleus to the cytoplasm. Nat Rev Mol Cell Biol 2007; 8: 761-773.

9. Calado A, Treichel N, Muller EC, Otto A, Kutay U. Exportin-5-mediated nuclear export of eukaryotic elongation factor 1A and tRNA. EMBO J 2002; 21: 6216-6224.

10. Liu G, Grant WM, Persky D, Latham VM Jr., Singer RH, Condeelis J. Interactions of elongation factor 1 alpha with F-actin and beta-actin mRNA: implications for anchoring mRNA in cell protrusions. Mol Biol Cell 2002; 13: 579-592.

11. Amiri A, Noei F, Jeganathan S, Kulkarni G, Pinke DE, Lee JM. eEF1A2 activates Akt and stimulates Akt-dependent actin remodeling, invasion and migration. Oncogene 2007; 26 : 3027-3040.

12. Murray JW, Edmonds BT, Liu G, Condeelis J. Bundling of actin filaments by elongation factor 1 alpha inhibits polymerization at filament ends. J Cell Biol 1996; 135: 1309-1321.

13. Duttaroy A, Bourbeau D, Wang XL, Wang E. Apoptosis rate can be accelerated or decelerated by overexpression or reduction of the level of elongation factor-1 alpha. Exp Cell Res 1998; 238: 168-176.

14. Lamberti A, Longo O, Marra M, Tagliaferri P, Bismuto E, Fiengo A et al. C-Raf antagonizes apoptosis induced by IFN-alpha in human lung cancer cells by phosphorylation and increase of the intracellular content of elongation factor 1A. Cell Death Differ 2007; 14: 952-962.

15. Mateyak MK, Kinzy TG. eEF1A: thinking outside the ribosome. J Biol Chem 2010; 285: 21209-21213.

16. Foster JL, Garcia JV. HIV-1 Nef: at the crossroads. Retrovirology 2008; 5: 84.

17. Ross TM, Oran AE, Cullen BR. Inhibition of HIV-1 progeny virion release by cell-surface CD4 is relieved by expression of the viral Nef protein. Curr Biol 1999; 9: 613-621.

18. Kirchhoff $F$. Immune evasion and counteraction of restriction factors by HIV-1 and other primate lentiviruses. Cell Host Microbe 2010; 8: 55-67.
19. Collins KL, Chen BK, Kalams SA, Walker BD, Baltimore D. HIV-1 Nef protein protects infected primary cells against killing by cytotoxic T lymphocytes. Nature 1998; 391: 397-401.

20. Geleziunas R, Xu W, Takeda K, Ichijo H, Greene WC. HIV-1 Nef inhibits ASK1-dependent death signalling providing a potential mechanism for protecting the infected host cell. Nature 2001; 410: 834-838.

21. Wolf D, Witte V, Laffert B, Blume K, Stromer E, Trapp S et al. HIV-1 Nef associated PAK and PI3-kinases stimulate Akt-independent Bad-phosphorylation to induce anti-apoptotic signals. Nat Med 2001; 7: 1217-1224.

22. Olivetta $E$, Federico M. HIV-1 Nef protects human-monocyte-derived macrophages from HIV-1-induced apoptosis. Exp Cell Res 2006; 312: 890-900.

23. Herbein G, Gras G, Khan KA, Abbas W. Macrophage signaling in HIV-1 infection. Retrovirology 2010; 7: 34.

24. Mahlknecht U, Ottmann OG, Hoelzer D. Far-Western based protein-protein interaction screening of high-density protein filter arrays. J Biotechnol 2001; 88: 89-94.

25. Cimarelli A, Luban J. Translation elongation factor 1-alpha interacts specifically with the human immunodeficiency virus type 1 Gag polyprotein. J Virol 1999; 73: 5388-5401.

26. Alessandrini L, Santarcangelo AC, Olivetta E, Ferrantelli F, d'Aloja P, Pugliese K et al. Ttropic human immunodeficiency virus (HIV) type 1 Nef protein enters human monocytemacrophages and induces resistance to HIV replication: a possible mechanism of HIV T-tropic emergence in AIDS. J Gen Virol 2000; 81: 2905-2917.

27. Li J, Yuan J. Caspases in apoptosis and beyond. Oncogene 2008; 27: 6194-6206.

28. Guo H, Tittle TV, Allen H, Maziarz RT. Brefeldin A-mediated apoptosis requires the activation of caspases and is inhibited by Bcl-2. Exp Cell Res 1998; 245: 57-68.

29. Talapatra S, Wagner JD, Thompson CB. Elongation factor-1 alpha is a selective regulator of growth factor withdrawal and ER stress-induced apoptosis. Cell Death Diff 2002; 9: 856-861.

30. Kobayashi $\mathrm{Y}$, Yonehara S. Novel cell death by downregulation of eEF1A1 expression in tetraploids. Cell Death Diff 2009; 16: 139-150.

31. Mei $Y$, Yong J, Liu H, Shi $Y$, Meinkoth J, Dreyfuss $G$ et al. tRNA binds to cytochrome $c$ and inhibits caspase activation. Mol Cell 2010; 37: 668-678.

32. Echarri A, Gonzalez ME, Carrasco L. The N-terminal Arg-rich region of human immunodeficiency virus types 1 and 2 and simian immunodeficiency virus Nef is involved in RNA binding. Eur J Biochem 1997; 246: 38-44.

33. Nissen P, Kjeldgaard M, Thirup S, Polekhina G, Reshetnikova L, Clark BF et al. Crystal structure of the ternary complex of Phe-tRNAPhe, EF-Tu, and a GTP analog. Science 1995; 270: 1464-1472.

34. Fackler OT, Baur AS. Live and let die: Nef functions beyond HIV replication. Immunity 2002; 16: 493-497.

35. Chuang SM, Chen L, Lambertson D, Anand M, Kinzy TG, Madura K. Proteasomemediated degradation of cotranslationally damaged proteins involves translation elongation factor 1A. Mol Cell Biol 2005; 25: 403-413.

36. Hotokezaka Y, Tobben U, Hotokezaka H, Van Leyen K, Beatrix B, Smith DH et al. Interaction of the eukaryotic elongation factor $1 \mathrm{~A}$ with newly synthesized polypeptides. $J$ Biol Chem 2002; 277: 18545-18551.

37. Nicchitta CV. Cell biology: How to combat stress. Nature 2009; 457: 668-669.

38. Varin A, Manna SK, Quivy V, Decrion AZ, Van Lint C, Herbein G et al. Exogenous Nef protein activates NF-kappa B, AP-1, and C-Jun N-terminal kinase and stimulates HIV transcription in promonocytic cells. Role in AIDS pathogenesis. J Biol Chem 2003; 278: 2219-2227.

39. Emiliani S, Fischle W, Van Lint C, Al-Abed Y, Verdin E. Characterization of a human RPD3 ortholog, HDAC3. Proc Natl Acad Sci USA 1998; 95: 2795-2800.

40. Livak KJ, Schmittgen TD. Analysis of relative gene expression data using real-time quantitative PCR and the 2(-Delta Delta C(T)) Method. Methods 2001; 25: 402-408.

Cell Death and Disease is an open-access journal published by Nature Publishing Group. This work is licensed under the Creative Commons Attribution-NonCommercial-No Derivative Works 3.0 Unported License. To view a copy of this license, visit http://creativecommons.org/licenses/by-nc-nd/3.0/

Supplementary Information accompanies the paper on Cell Death and Disease website (http://www.nature.com/cddis) 


\section{Retraction}

\section{Inhibition of ER stress-mediated apoptosis in macrophages by nuclear-cytoplasmic relocalization of eEF1A by the HIV-1 Nef protein}

W Abbas, KA Khan, MK Tripathy, I Dichamp, M Keita, O Rohr and G Herbein

Cell Death and Disease (2012) 3, e368; doi:10.1038/cddis.2012.104; published online 9 August 2012

Retraction to: Cell Death and Disease (2012) 3, e292; doi:10.1038/cddis.2012.32; published online 5 April 2012.

The authors agree to retract the above article in Cell Death and Disease 2012 due to the use of a mismatched image in Figure 1a first shown in the Journal of Biotechnology, 2001, 88: 89-94. An active collaboration between the research groups of the two above articles occurred in the late nineties.
Unfortunately this image shared 10 years ago as a far-western blot example was misunderstood to be the actual far-western blot for HIV-1 Nef as a bait and published in Cell Death and Disease 2012 article. The authors stand behind data showing that Nef by binding to EF1A inhibits ER stressmediated apoptosis in macrophages. They apologize for any inconvenience caused. 